\title{
Strategic capacity sharing between two tasks: evidence from tasks with the same and with different task sets
}

\author{
Carola Lehle $\cdot$ Ronald Hübner
}

\begin{abstract}
The goal of the present study was to investigate the costs and benefits of different degrees of strategic parallel processing between two tasks. In a series of experiments with the dual-task flanker paradigm, participants were either instructed to process the tasks serially or in parallel, or - in a control condition-they received no specific instruction. Results showed that the participants were able to adjust the degree of parallel processing as instructed in a flexible manner. Parallel processing of the two tasks repeatedly led to large costs in performance and to high crosstalk effects compared to more serial processing. In spite of the costs, a moderate degree of parallel processing was preferred in the condition with no specific instruction. This pattern of results was observed if the same task set was used for the two tasks, but also if different ones were applied. Furthermore, a modified version of the central capacity sharing (CCS) model (Tombu and Jolicoeur in $\mathbf{J}$ Exp Psychol Hum Percept Perform 29:3-18, 2003) was proposed that accounts also for crosstalk effects in dual tasks. The modified CCS model was then evaluated by fitting it successfully to the present data.
\end{abstract}

C. Lehle $(\varangle)$

Biologische Psychologie/Psychophysiologie,

Humboldt-Universität zu Berlin, Rudower Chausee 18,

12489 Berlin, Germany

e-mail: carola.lehle@cms.hu-berlin.de

R. Hübner

Universität Konstanz, Constance, Germany

\section{Introduction}

The question of how efficiently humans can perform more than one task at a time is of major interest for theoretical as well as for practical reasons. Accordingly, dual-task studies have a long tradition in experimental psychology (for a review see, e.g., Pashler, Johnston, \& Ruthruff, 2001). Dual-task processing is usually accompanied by costs compared to a situation where the tasks are conducted separately (Logan \& Gordon, 2001; Logan \& Schulkind, 2000; Pashler \& Johnston, 1989).This indicates that the ability of parallel processing in the human information processing system is limited.

Several issues about serial and parallel processing in dual tasks, however, are still unresolved. First, it has to be clarified whether a strategy of serial or of parallel processing is more advantageous in dual tasks. It is frequently assumed that a serial processing strategy is favorable in dual tasks, because it minimizes confusion and crosstalk (Logan \& Gordon, 2001; Navon \& Miller, 1987; Tombu \& Jolicoeur, 2003). Other researchers assume that participants prefer a parallel processing strategy-at least at short stimulus onset asynchronies (Miller, Ulrich, \& Rolke, 2008).

In a previous study (Hübner \& Lehle, 2007), we investigated processing strategies in single and dual tasks and applied a combination of the Eriksen Flanker task with the psychological refractory period (PRP) paradigm (Telford, 1931). There, participants had to make a first speeded response $\mathrm{R} 1$ to a centrally presented target stimulus (S1). Then, while the processing of the first task was still in progress, flanker stimuli (S2) were presented with a variable stimulus onset asynchrony (SOA), for which a second response R2 was conducted. Because the same judgment type was required for $S 1$ and $S 2$, the stimuli were 
congruent or incongruent, that is associated with the same response category or not. As a main result, the participants showed a strong tendency to process the dual tasks in parallel, although it could be shown that they were also able to process them serially. The flanker congruency effect (FCE), i.e., the result that the latencies were increased in incongruent compared to congruent situations, was substantially increased in dual compared to single tasks. In a series of experiments (Hübner \& Lehle, 2007), the FCE appeared to reliably indicate the degree of strategic parallel processing. If participants adopted a strict serial strategy in the dual tasks, the FCE was absent. Furthermore, the results suggested that increased parallel processing did not lead to a benefit in performance, but to costs on RT1. Apart from that, the PRP effect, i.e., the result that the response time for the second task (RT2) increases with decreasing SOA, was largely unaffected by the degree of parallel processing.

In the previous study, however, not only the degree of parallel processing, but also the methods changed considerably between the experiments. This probably confounded the comparison of different degrees of parallel processing between two tasks. Moreover, the same judgment type was used for each task which might have restricted the generality of the results. Therefore, in the present study, we intended to evaluate the consequences of strategic serial and parallel processing in dual tasks more unequivocally.

Moreover, up to now, it is not clear which model accounts best for the strategic variation of serial to parallel. processing in dual tasks. The most prominent explanation of the PRP effect is provided by the central bottleneck (CB) model (Pashler, 1984; Pashler \& Johnston, 1989), which assumes that each task must pass three serial stages: Perception, response selection, and response execution. Whereas perception and response execution can proceed in parallel for two tasks, it is supposed that the central response selection stage is restricted to one task at a time. Thus, according to the CB model, the PRP effect is due to the queuing of the second task at this bottleneck. The earlier S2 arrives, the longer the second task has to wait at the central stage for being processed.

Similar to the CB model, most advocates of capacity sharing models propose that capacity on a central stage is limited. However, different to the CB model, they assume that capacity can be shared at all stages of processing and that the degree of serial to parallel processing in dual tasks can be adjusted deliberately (Gopher, 1986; Kahneman, 1973; McLeod, 1977). In the central capacity sharing (CCS) model, substantial progress has been made in formalizing the capacity sharing idea (Navon \& Miller, 2002; Tombu \& Jolicoeur, 2003). Costs in dual tasks such as the PRP effect do not imply strict serial processing; they can also be explained by assuming a capacity limitation on the central stage ${ }^{1}$ (for a comprehensive review see Navon \& Miller, 2002). Furthermore, CCS theories might account for results difficult to reconcile with the all-or-none bottleneck idea (Ellenbogen \& Meiran, 2008).

The CB model can be conceptualized as a special case of the CCS model, because the predictions of both models are identical if, during the first task, all capacity is allocated on T1. Because the overall amount of capacity remains fixed, RT1 is predicted to increase the more of the capacity is allocated to T2. At the same time, RT2 and the PRP effect should be unaffected by varying degrees of parallel processing (for details of the CCS model see "Appendix 1"). As already pointed out, the prediction that increased parallel processing affects mainly RT1 seemed to be supported by the results of our previous study (Hübner \& Lehle, 2007), although definite conclusions were not possible.

The aim of the present study was to evaluate the costs and benefits of a serial and parallel processing strategy in dual tasks and thus also the formal predictions of the CCS model more unequivocally. For this objective, different degrees of parallel processing were realized by instructing the participants accordingly, while the essential features of the tasks remained fixed. The experiments were again a combination of the Eriksen Flanker task with the PRP paradigm similar to our previous study (Hübner \& Lehle, 2007). Furthermore, in order to see whether the results hold more generally, also experiments with different stimuli and judgment types for the two tasks were conducted.

Apart from that, however, there is still the problem of how congruency effects in dual tasks can be integrated in the original $\mathrm{CB}$ or in the $\mathrm{CCS}$ model (for crosstalk effects in dual tasks see also, e.g., Fischer, Miller, \& Schubert, 2007; Lien \&. Proctor, 2002; Lien, Schweickert, \& Proctor, 2003; Logan \& Gordon, 2001; Logan \& Schulkind, 2000; Miller, 2006; Miller \& Alderton, 2006). For the CB model, a modified version has been proposed where it is assumed that $R 1$ and $R 2$ can be activated in parallel, thus explaining the crosstalk effects, whereas the final selection of a response occurs strictly serial (Hommel, 1998; Lien \& Proctor, 2002; Lien et al., 2003). For the CCS model

\footnotetext{
We define central capacity similar to the capacity of cognitive control by Lavie, Hirst, De Fockert, \& Viding (2004): it encompasses postperceptual operations associated with higher cognitive functions which have been demonstrated to impose a large decline in performance when conducted concurrently with other operations. Besides response selection (Pashler, 1994a), these operations likely encompass also memory consolidation (e.g., Jolicoeur \& Dell'Acqua, 1998), mental rotation (Band \& Miller, 1997), suppression of response priming (Stürmer, Seiss, \& Leuthold, 2005) and presumably also difficult conditions of stimulus selection (e.g., Jolicoeur et al, 2006; Magen \& Cohen, 2005). There is definitively a need for further clarification, respectively, integration or dissociation of the different operations. However, this is not at the scope of the present study.
} 
(Tombu \& Jolicoeur, 2003), an extension will be proposed so that it can also be applied to situations where crosstalk effects arise. The extended CCS model will then be evaluated by fitting it to our data. Finally, its explanatory power in contrast to other dual task models will be discussed.

To sum up, the main goal of the present study was to compare the costs and benefits of serial and parallel processing in two tasks that are congruent or incongruent to each other. In Experiments 1A and 1B, two tasks with the same task set were used, whereas the task set changed between Task 1 and Task 2 in Experiments $2 \mathrm{~A}$ and $2 \mathrm{~B}$. Participants were instructed to process the dual tasks serially or in parallel in Experiments $1 \mathrm{~A}$ and $2 \mathrm{~A}$. In Experiments $1 \mathrm{~B}$ and 2B, no specific instruction was given in order to investigate which strategy is spontaneously adopted by the participants. The obtained results were then used to evaluate the CCS idea more thoroughly and to extend the CCS model so that it is also applicable to dual tasks with crosstalk.

Apart from that, also dynamic aspects of the dual-task processing strategies were considered in the present study. On the one hand, we examined whether our participants were able to switch between serial and parallel processing within an experimental session. Moreover, we used a specific procedure to reveal the dynamic modulation within a trial: on half of the trials, the flankers changed their identity after a short time interval. On these Alteringflanker trials, the initial flankers (First-part flankers) were always irrelevant for the task, whereas the second flankers (Second-part flankers) had to be responded to after the response to the target. The Second-part flankers appeared in a different color so that they could easily be identified. On the other half of the trials, the Fixed-flanker trials, only the color of the stimuli changed as on the Altering-flanker trials, but their identity remained constant. ${ }^{2}$ The presentation of Altering- and Fixed-flanker trials was randomly intermixed.

Because the irrelevant First-part flankers were also either congruent or incongruent to the target, the degree of their coprocessing should be reflected in a corresponding congruency effect: In case of strict serial processing, they should have no effect on RT1 or on RT2. In case of parallel processing, however, it can be expected that the First-part flankers lead to a congruency effect-at least on RT1.

\footnotetext{
${ }^{2}$ That we included the particular proportion of $50 \%$ Altering-flanker and 50\% Fixed-flanker trials had the following reason: In a dual-task experiment where flanker stimuli changed on every trial, participants adopted a strategy of very serial processing (Hübner \& Lehle, 2007; Experiment 4). In contrast, if the flankers did not change, a parallel strategy was preferred (Hübner \& Lehle, 2007, Experiments 2A, B, C). Thus, by mixing Altering-flanker and Fixed-flanker trials equally, we intended to create a condition that itself neither induces an extreme parallel nor an extreme serial processing strategy.
}

Apart from that, since the First-part flankers were irrelevant, it was also possible that the degree of flanker processing is modulated within a trial, i.e., between the First- and the Second-part flankers. Importantly, because no response was required to the First-part flankers, their effects cannot be attributed to a strategy of response grouping or of merely delaying the response.

\section{Experiment 1A}

The aim of Experiment $1 \mathrm{~A}$ was to compare serial and parallel processing strategies in dual tasks with the same judgment type. This was realized by instructing the participants accordingly.

After the results had shown that our processing instructions had the intended effects, we ran Experiment $1 \mathrm{~B}$ with the same conditions as in Experiment 1A, except that no specific processing instruction was given. The question was what degree of capacity sharing participants would choose without a specific instruction. The method and the results of Experiment $1 \mathrm{~B}$ are reported directly after the Results section of Experiment 1A. Both experiments are then discussed together.

Method

\section{Participants}

A total of 12 students ( 6 males, 6 females) participated in this study either for partial fulfillment of course requirements or for getting paid $5 \mathrm{\epsilon} / \mathrm{h}$. Their age ranged from 20 to 30 years ( $M=24$ years). All were right-handed (by self report), and had normal or corrected-to-normal vision.

\section{Apparatus}

The stimuli were presented on a 21 -in. color monitor with a resolution of $1,280 \times 768$ pixels, and a refresh rate of $85 \mathrm{~Hz}$. A personal computer (PC) served for controlling stimulus presentation and response registration.

\section{Stimuli}

The stimulus set consisted of the numerals $(1,2,3,4,6$, 7,8 , and 9). The height of the stimuli subtended a visual angle of $2^{\circ}$ at a viewing distance of $110 \mathrm{~cm}$, and their width was about $1.36^{\circ}$, depending on the specific digit. The target (S1) was presented at the center of the screen, whereas the flankers (S2) consisted of two copies of a numeral symbol, which were presented left and right of $\mathrm{S} 1$ at an eccentricity of $1.18^{\circ}$. Target and flankers were always different. 


\section{Procedure}

The task for the participants was to judge the parity (ODD, EVEN) of the stimuli. They had to press a left key for EVEN and a right key for ODD. Each trial started with the appearance of a fixation cross for $400 \mathrm{~ms}$. After a blank screen of $600 \mathrm{~ms}$ duration, both target and flankers were presented in white color on a black background. After a variable time interval of 50,150 , or $350 \mathrm{~ms}$, the color of the stimuli changed to red or green. The color of the flankers was always different to that of the target. On half of the trials (Altering-flanker trials), the flankers also altered their identity with coloring, i.e., the originally appearing flankers (First-part flankers) were replaced by new numerals (Second-part flankers) after the respective time interval. The interval until the onset of the Secondpart flankers is called First-part interval. The First-part flankers were irrelevant, whereas the Second-part flankers had to be responded to in Task 2. On the other half of the trials (Fixed-flanker trials), the identity of the flankers remained constant, but their color changed after the time interval as in the Altering-flanker trials. This interval in the Fixed-flanker trials, to avoid confusion, is called First-color interval. The Altering- and the Fixed-flanker trials were presented in random order, so that a specific preparation was not possible.

Flankers were congruent on half of the trials, i.e., had the same parity as the target, and were incongruent on the other half, i.e., had the opposite parity as the target. In Alteringflanker trials, the congruency could change from First-part to Second-part flankers. Thus, there were four different congruency combinations, which were equal in frequency and randomized across trials. The dependent measures were the latencies and error rates of both responses.

Participants always had to respond (R1) to the target first by pushing one of two buttons with the left hand, and subsequently respond (R2) to the (Second-part) flankers by pushing one of two other buttons with the right hand. The stimuli remained on the screen until the participants' responses had occurred. One second after the last response, the cue for the next trial appeared. Errors for R1 and R2 were signaled by individual tones.

Participants received specific instructions on how they had to allocate their capacity. There were two block types: For one type, the participants were instructed to allocate their capacity first to the target only and to start flanker processing not before the selection of R1. For the other block type, the participants were instructed to distribute their capacity also to the flankers right from the beginning of a trial. Four successive blocks of one instruction type alternated with four blocks of the other type. Altogether there were 16 blocks with 96 trials each, which were distributed to two separate 1-h sessions. In the first session, there was also a corresponding training block before each specific instruction section. ${ }^{3}$ The instruction order was balanced across participants.

\section{Results}

\section{Response times}

RTI In a first step, the latencies of correct responses to S1 were analyzed by an overall two-factor analysis of variance (ANOVA) for repeated measurements on the factors Instruction (serial, or parallel), and Flanker (fixed, or altering). Because the other factors differed between the two flanker conditions, these factors were analyzed separately for the two flanker conditions.

The overall analysis revealed a significant main effect of Instruction, $F(1,11)=91.4, p<0.001$. As can be seen in Fig. 1, participants responded $238 \mathrm{~ms}$ faster under the serial (left panels) than under the parallel instruction (center panels). Furthermore, the main effect of Flanker was reliable, $F(1,11)=64.3, p<0.001$. Latencies were $73 \mathrm{~ms}$ longer on Altering-flanker trials than on Fixedflanker ones. However, there was also a significant interaction between Instruction and Flanker, $F(1,11)=9.37$, $p<0.05$. The effect of the altering flankers was larger under the parallel than under the serial instruction.

Fixed flanker The data for the Fixed-flanker trials (see Fig. 1, upper panels) were entered into a three-factor ANOVA on the factors Instruction (serial, or parallel), Congruency (congruent, or incongruent), and First-color interval $(50,150$, or $350 \mathrm{~ms})$. The analysis revealed a significant main effect of Instruction, $F(1,11)=107$, $p<0.001$. Latencies were shorter under the serial than under the parallel instruction (697 vs. $916 \mathrm{~ms}$ ). Also Congruency had a significant main effect of $179 \mathrm{~ms}$, $F(1,11)=135, p<0.001$. However, there was a significant interaction between Instruction and Congruency, $F(1,11)=94.0, p<0.001$. The congruency effect was smaller under the serial than under the parallel instruction (70 vs. 289 ms; see Fig. 1, left compared to center panels). Further tests revealed that, although Instruction had a larger effect in the incongruent condition, its effect was also significant in the congruent condition, $t(11)=7.97$, $p<0.001$. Finally, the effect of First-color interval was significant, $F(2,22)=23.8, p<0.001$. The latencies increased at the longest First-color interval, compared to the other two intervals (790, 788, and $842 \mathrm{~ms}$ ).

\footnotetext{
3 To analyze practice effects, we conducted several ANOVAs for the two experiments and the different instruction conditions-containing block number as a factor. There, we revealed always a main effect of block number, i.e., the response times (RT1 and RT2) decreased with increasing practice. However, there was no significant interaction of block and congruency.
} 
Fig. 1 RTl data for Experiments $1 \mathrm{~A}$ and $1 \mathrm{~B}$. The upper panels show the data of the Fixed-flanker trials. The lower panels depict the data of the Altering-flanker trials, in dependence of First-part and Second-part flanker congruency. "Con" and "inc" denote congruent and incongruent, respectively. Effects of Secondpart flanker congruency are expressed by the differences between the filled symbols (congruent) and unfilled symbols (incongruent), whereas First-part FCEs can be identified by comparing the different data points with filled, respectively, unfilled symbols in the lower panels
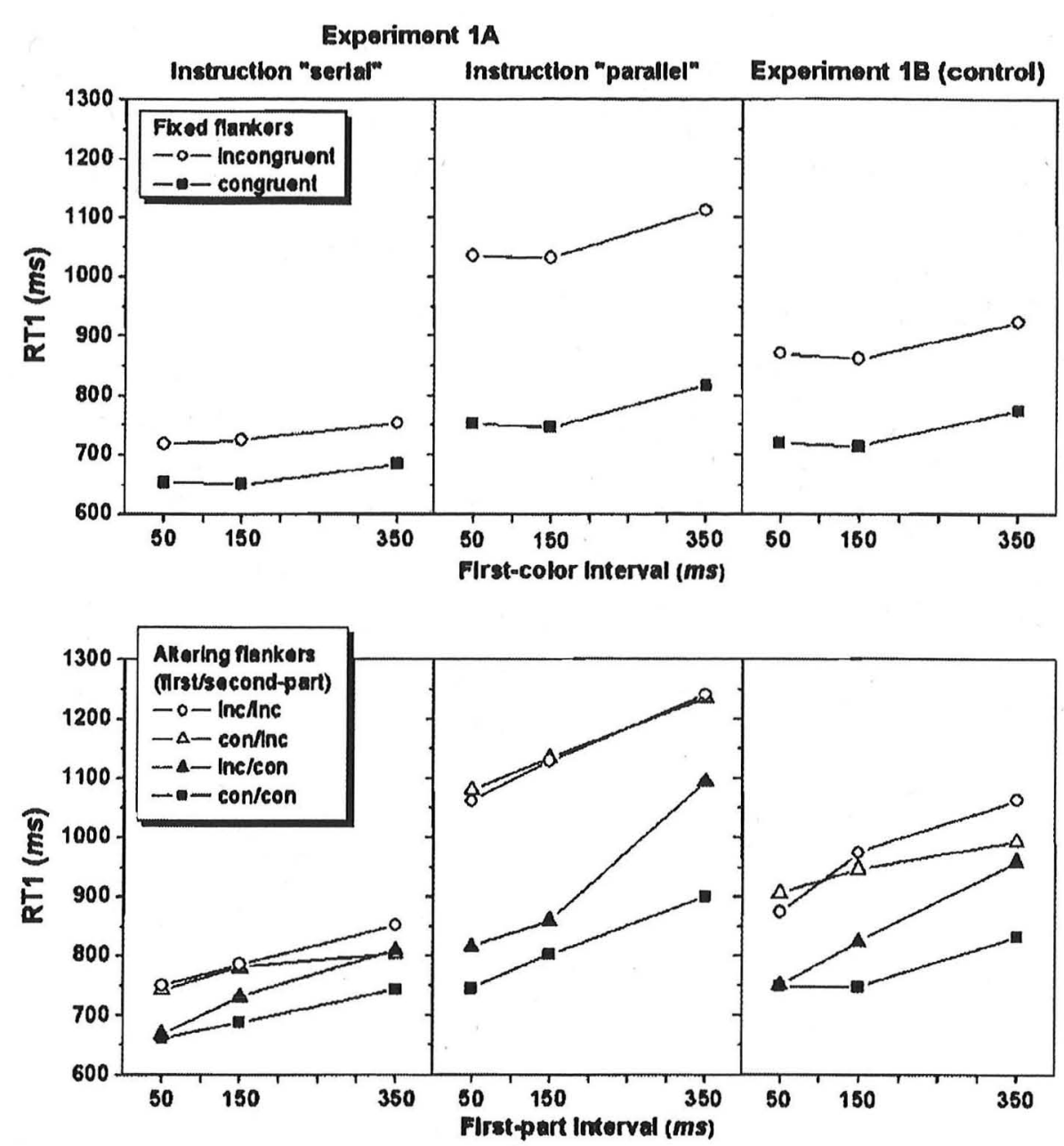

Altering flanker The data for the Altering-flanker trials (see Fig. 1, lower panels) were entered into a four-factor ANOVA on the factors Instruction (serial, or parallel), First-part congruency (congruent, or incongruent), Secondpart congruency (congruent, or incongruent), and First-part interval $(50,150$, or $350 \mathrm{~ms})$. The analysis revealed a significant main effect of Instruction, $F(1,11)=75.8$, $p<0.001$. Latencies under the serial instruction were shorter than those under the parallel instruction (751 vs. $1,008 \mathrm{~ms})$. Furthermore, the main effect $(40 \mathrm{~ms})$ of Firstpart congruency was reliable, $F(1,11)=16.0, p<0.01$, as was the main effect (173 ms) of Second-part congruency, $F(1,11)=64.1, p<0.001$.

However, there was also a significant interaction between First-part and Second-part congruency, $F(1,11)=12.8$, $p<0.01$. As can be seen in Fig. 1, First-part congruency had a substantial effect only when the second-part flankers were congruent. Second-part congruency also interacted reliably with Instruction, $F(1,11)=39.1, p<0.001$. The congruency effect was smaller under the serial than under the parallel instruction ( 69 vs. $277 \mathrm{~ms}$ ). As a further test revealed, Instruction had also a significant effect in the congruent condition, $t(11)=6.98, p<0.001$.

Also First-part interval had a significant main effect, $F(2,22)=37.7, p<0.001$. Latencies were 815,863 , and $959 \mathrm{~ms}$ for the three-First-part intervals, respectively. Furthermore, First-part interval interacted significantly with First-part congruency, $F(2,22)=6.92, p<0.01$, and with Second-part congruency, $F(2,22)=8.38, p<0.01$. The First-part congruency effects increased with the duration of the First-part interval $(17,25$, and $79 \mathrm{~ms})$. The effects for the Second-part flankers were 186, 187, and $146 \mathrm{~ms}$ for the First-part intervals of 50,150, and $350 \mathrm{~ms}$, respectively. First-part interval also interacted with Instruction, $F(2,22)=17.3, p<0.001$. Under the serial instruction, the increase of latencies at a First-part interval of $350 \mathrm{~ms}$ compared to $50 \mathrm{~ms}$ was smaller than under the parallel instruction (96 vs. $192 \mathrm{~ms}$ ).

Finally, there was a four-way interaction between all factors, $F(2,22)=4.25, p<0.05$. As can be seen in 
Fig. 1, this effect indicates that the two-way interaction between the two congruency factors was more pronounced under the parallel instruction, and especially for the longest First-part interval.

RT2 RT2 is usually measured from the onset of S2. Here, the flankers always appeared simultaneously with S1. However, we have to take our specific temporal variation procedure into account. In the Fixed-flanker condition, we measured the latencies beginning from stimulus onset. For the Altering-flanker condition, though, the relevant flankers appeared with a delay. In this case, the latencies should be measured from the onset of the Second-part flankers. However, the result that the First-part flankers affected RT1 suggests that they also affected RT2. To see whether this was the case, the latencies for the Altering-flanker condition were measured from the onset of the Second-part flankers, but the analysis included the congruency of the First-part flankers as a factor. In Fig. 2, the data were plotted accordingly.
Fixed flanker Latencies of the correct responses (for RI and R2) on Fixed-flanker trials (see Fig. 2, upper panels) were entered in a three-factor ANOVA for repeated measures on the factors Instruction (serial, or parallel), Congruency (incongruent, or congruent), and First-color interval $(50,150$, or $350 \mathrm{~ms})$. The analysis revealed significant main effects of Instruction (79 ms), $F(1,11)=15.9$, $p<0.01$, and of Congruency $(237 \mathrm{~ms}), F(1,11)=174$, $p<0.001$. However, there was also a significant interaction between these two factors, $F(1,11)=110, p<0.001$. The congruency effects were 123 and $350 \mathrm{~ms}$ for the serial (see Fig. 2, left panels) and parallel instructions (center panels), respectively. Contrary to $\mathrm{RT} 1$, the instruction effect was significant for the incongruent condition, $t(11)=7.62$, $p<0.001$, but not for the congruent one, $t(11)=0.102$, $p=0.102$. Finally, also First-color interval produced a significant effect, $F(2,22)=22.8, p<0.001$. Latencies increased by $62 \mathrm{~ms}$ over the First-color interval range.

Altering flanker The data for correct responses ( $R 1$ and R2) of the Altering-flanker trials (see Fig. 2, lower panels)
Fig. 2 RT2 data for Experiments $1 \mathrm{~A}$ and $1 \mathrm{~B}$. The upper panels show the data of the Fixed-flanker trials. The lower panels depict the data of the Altering-flanker trials, in dependence of First-part and Second-part flanker congruency. Note that for the Alteringflanker trials, RT2 was measured from the onset of the Second-part flankers. "Con" and "inc" denote congruent and incongruent, respectively. Effects of Second-part flanker congruency are expressed by the differences between the filled symbols (congruent) and unfilled symbols (incongruent), whereas First-part FCEs can be identified by comparing the different data points with filled. respectively, unfilled symbols in the lower panels
Experiment 1A

were entered in a four-factor ANOVA for repeated measures on the factors Instruction (serial, or parallel), Firstpart congruency (incongruent, or congruent), Second-part congruency (incongruent, or congruent), and First-part interval $(50,150$, or $350 \mathrm{~ms})$. The analysis revealed a significant main effect of Instruction, $F(1,11)=29.0$, $p<0.001$. RT2 increased by $115 \mathrm{~ms}$ under the parallel instruction relative to the serial instruction. Also the main effects of First-part congruency, $F(1,11)=11.7$, $p<0.01$, and of Second-part congruency, $F(1,11)=119$, $p<0.001$, were significant. Similar as for RT1, there was also a reliable interaction between the two congruency factors, $F(1,11)=38.8, p<0.001$ (see Fig. 2).

First-part congruency also interacted with Instruction, $F(1,11)=11.7, p<0.01$, as did Second-part congruency, $F(1,11)=35.6, p<0.001$. In each case the congruency effect was smaller under the serial instruction than under the parallel instruction (First-part congruency: 15 vs. $50 \mathrm{~ms}$; Second-part congruency: 116 and $337 \mathrm{~ms}$ ). The significant three-way interaction between Instruction and the two congruency factors, $F(1,11)=9.69, p<0.01$, indicated that Instruction had a larger impact on Second-part congruency than on First-part congruency. Concerning the interaction between Second-part congruency and Instruction, however, it is important to note that Instruction had a significant effect for the incongruent condition, $t(11)=6.94, p<0.001$, but not for the congruent one, $t(11)=0.210, p=0.836$.

Furthermore, there was a significant main effect of Firstpart interval, $F(2,22)=28.6, p<0.001$, which indicates a PRP effect. RT2 was 984,941 , or $884 \mathrm{~ms}$ for the first-part intervals of 50,150 , or $350 \mathrm{~ms}$, respectively. However, the first-part interval effect was qualified by significant two-way interactions with Instruction, $F(2,22)=11.2, p<0.001$, with First-part congruency, $F(2,22)=6.67, p<0.01$, and with Second-part congruency, $F(2,22)=5.96, p<0.01$. It appears that the effect of First-part congruency increased with the duration of the First-part interval $(15,17$, and $68 \mathrm{~ms}$ ), whereas it decreased for Second-part congruency $(240,245$, and $196 \mathrm{~ms})$. This difference produced a significant three-way interaction between First-part interval and the two congruency factors, $F(2,22)=12.4, p<0.001$.

Finally, there was a significant four-way interaction between all factors, $F(2,22)=5.750, p<0.01$. As can be seen in Fig. 2, this interaction was obviously due to the same reasons as the corresponding interaction for RT1.

\section{Error rates}

The mean error rate for $\mathrm{R} 1$ was $3.37 \%$ under the serial, and $4.87 \%$ under the parallel instruction. The error rate for $\mathrm{R} 2$, under the condition that $\mathrm{R} \mathbf{l}$ had also been correct, averaged to $4.91 \%$ under the serial, and $3.70 \%$ under the parallel instruction. Because the error rates were rather low and did not indicate any speed-accuracy trade-off, they are not further reported here.

\section{Experiment 1B}

This experiment served as control condition for Experiment 1A. The same procedure was applied as in Experiment 1A, except that now no specific processing instruction was given. It should thus be examined which processing strategy would be adopted spontaneously by the participants.

\section{Method}

A total of 12 students ( 7 females, 5 males) participated in this study under similar conditions as in the previous experiment. Their age ranged from 20 to 30 years ( $M=24.3$ years). All were right-handed (by self report), had normal or corrected-to-normal vision, and none had participated in Experiment 1A. The stimuli and procedure were the same as in Experiment 1A. Here, however, no specific instruction was given concerning the allocation of capacity to the tasks. Participants were merely informed about the tasks and the response order.

\section{Results}

\section{Response times}

The main difference to Experiment 1A was that the results of the present experiment were now rather in-between the two instruction conditions of Experiment 1A. Apart from that, the data pattern-in particular the temporal effects and the interactions-were very similar to Experiment $1 \mathrm{~A}$. Because of that, only the overall results from the analyses will be reported here.

RTI As in Experiment 1A, an overall ANOVA was computed first, including the correct responses for the Fixed-flanker and the Altering-flanker trials on the factors Flanker (fixed, or altering) and Congruency (congruent, or incongruent). As a significant main effect of Flanker, $F(1,11)=64.5, p<0.001$, latencies averaged to 884 and $810 \mathrm{~ms}$ for Altering and Fixed-flanker trials (see Fig. 1, right panels). Furthermore, there was a significant main effect of (Second-part) Congruency (149 ms), $F(1,11)=$ $29.4, p<0.001$. Apart from that, a separate ANOVA on the Altering-flanker trials revealed a significant main effect of First-part congruency $(45 \mathrm{~ms}), \quad F(1,11)=29.6$, $p<0.001$.

RT2 The latencies of correct responses for Fixed-flankers and for Altering-flankers trials were entered in two separate 
ANOVAs for repeated measurements. They revealed a significant congruency effect $(192 \mathrm{~ms})$ on the Fixed-flanker trials, $F(1,11)=37.8, p<0.001$, as well as significant main effects of First-part congruency (47 ms), $F(1,11)=59.9, p<0.001$, and of Second-part congruency $(203 \mathrm{~ms}), \quad F(1,11)=54.3, p<0.001$, on the Altering-flanker trials (see Fig. 2, right panels).

\section{Error rates}

The mean error rate for $\mathrm{R} 1$ was $4.62 \%$, and that for $\mathrm{R} 2$ was $4.94 \%$, given that $\mathrm{R} 1$ had also been correct. For the same reasons as in Experiment $1 \mathrm{~A}$, the data are not further reported.

\section{Discussion of experiments $1 \mathrm{~A}$ and $1 \mathrm{~B}$}

Our results show that the participants were indeed able to process the dual tasks in the instructed way. This is indicated by the FCE, which was substantially larger under the parallel than under the serial instruction. A further result was that RT1 was generally increased under the paralle] than under the serial condition, i.e., also on congruent trials. The situation was different for RT2. Compared to the serial condition, RT2 was increased only for incongruent flankers under the parallel instruction.

The mean performance in Experiment 1B was intermediate compared to the performance under the serial and the parallel instructions in Experiment 1A. That is, if no specific processing instruction is given, participants seem to adopt a medium degree of parallel processing between the dual tasks. ${ }^{4}$ By comparing the effects of the three conditions (see Fig. 3), it becomes apparent that parallel processing does not lead to a benefit in performance. In the incongruent condition, there were even large costs of the increased coprocessing for both RT1 and RT2. In the congruent condition, substantial costs could be observed for RT1, whereas RT2 was not different for the two instruction conditions.

Furthermore, also the irrelevant First-part flankers were coprocessed, as indicated by their congruency effects on both RT1 and RT2. That the First-part flankers also affected RT2 - and this even more with a parallel processing strategy-indicates that the information extracted from the First-part flankers remained even after the flankers were changed and R1 was executed. Apart from the congruency

\footnotetext{
4 The variance across subjects was rather high in all Experiments reported in the present study, which indicates that there was some between-subjects variability in the degree of serial or parallel processing that was applied in the dual tasks. However, the mean SD in Experiment 1B was not increased, but even smaller compared to Experiment $1 \mathrm{~A}$ (198 vs. $228 \mathrm{~ms}$ in RT1; 214 vs. $237 \mathrm{~ms}$ in RT2). This was also the case for Experiment $2 \mathrm{~B}$ compared to Experiment 2A (185 vs. $232 \mathrm{~ms}$ in RT1; 199 vs. $269 \mathrm{~ms}$ in RT2).
}

effects, the strategy of First-part flanker coprocessing also increased overall RT1 compared to the Fixed-flanker trials.

However, in Experiments 1A and 1B, the same judgment type was used for both tasks which might restrict the generality of the observed results. If the same judgment type is used, target and flankers activate the mental representation of categories such as ODD or EVEN, which are associated with response categories such as LEFT or RIGHT, depending on the specific stimulus-response mapping. Within such a structure, flankers can also activate the target categories according to the task set of Task 1. Although a number of studies indicate that the FCE is primarily due to crosstalk between response categories (for a review see, e.g., Cohen \& Shoup, 1997), crosstalk between stimulus categories cannot be excluded for the present experiments. Therefore, we conducted two further experiments that were analogous to Experiments $1 \mathrm{~A}$ and 1B, except that individual stimuli and judgment types were used for each task.

\section{Experiment 2A}

The same procedure as in Experiment $1 \mathrm{~A}$ was applied in Experiment 2A, except that the participants had to perform letter categorization (CONSONANT/VOWEL) for Task 2 . Accordingly, each task had its own set of stimulus categories and crosstalk could only occur between response categories (cf. Hübner \& Druey, 2006; Watter \& Logan, 2006). Recent evidence suggests that parallel activation of response categories is not only possible in dual tasks with the same task sets, but also with different ones (Fischer et al., 2007) depending on the availability of resources, i.e., the ease to which it can be switched between the two task sets (Oriet, Tombu, \& Jolicoeur, 2005).

Additionally, also neutral flankers were presented on $25 \%$ of the trials to compare the performance on these trials with that for Task 1 on congruent dual-task trials. In the present experiment, two different task sets were used for the target and the flankers. It has been demonstrated that, if stimuli associated with different task sets are presented in a task, interference might not only result from competition between individual response categories, but also from competition between task sets (Rogers \& Monsell, 1995; Steinhauser \& Hübner, 2007; Waszak, Hommel, \& Allport, 2003). By comparing the performance on trials with congruent and with neutral flankers, we intended to investigate the influence of the degree of parallel processing on the task set activation effect. Because the size of the task set conflict has been shown to depend on several factors such as the salience of the distractors, one can presume that increased parallel processing also leads to an increased task set activation.

Furthermore, we again conducted a control experiment (Experiment 2B) without a specific instruction, analogous 
Fig. 3 RT1 and RT2 data from Experiment $1 \mathrm{~A}$ (scrial, parallel) and Experiment $1 \mathrm{~B}$ (control). ${ }^{*} p<0.05$, *** $p<0.001$. All Fixed- and Altering-flanker trials are included. The RT2 data are shown as measured from onset of $\mathrm{SI}$

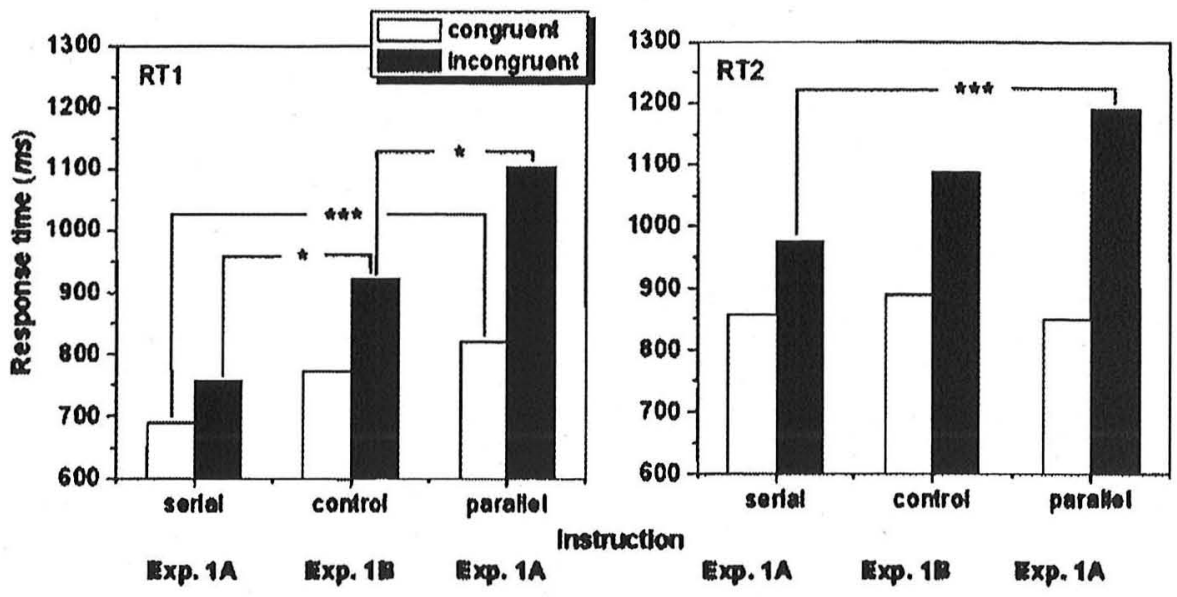

to Experiment 1B. As before, the results of Experiments $2 \mathrm{~A}$ and $2 \mathrm{~B}$ are discussed together in a common section.

Method

A total of 16 students (10 females, 6 males) participated in this study. Their age ranged from 20 to 29 years ( $M=23.6$ years). All were right-handed (by self report), had normal or corrected-to-normal vision, and none had participated in Experiment 1A or 1B. The procedure was the same as in Experiment 1A, with two exceptions. First, on $75 \%$ of the trials letters (A, E, G, I, K, M, R, U) were used as flankers (S2). On these trials, Task 2 was to judge whether the letter was a consonant or a vowel. A left key had to be pressed for VOWEL and a right key for CONSONANT. For $S 1$, numerals were used $(1,2,3,4,6,7,8$, and 9) that had to be judged for ODD or EVEN as in Experiments $1 \mathrm{~A}$ and $1 \mathrm{~B}$. Because of that, participants had to switch the task sets between $\mathrm{T} 1$ and $\mathrm{T} 2$. Second, on the remaining $25 \%$ of trials a neutral symbol (\#, or \%) was presented as flankers. On these trials no second response was required.

\section{Results}

\section{Response times}

RTI In a first step, the latencies of correct responses to S1 were analyzed by an overall ANOVA for repeated measurements on the factors Instruction (serial, or parallel), and Flanker (fixed, or altering). Because the other factors differed between the two flanker conditions, these factors and the data from the neutral condition were analyzed separately.

The overall analysis revealed a significant main effect of Instruction, $\quad F(1,15)=24.2, \quad p<0.001$. Participants responded $182 \mathrm{~ms}$ faster under the serial than under the parallel instruction. Furthermore, there was a significant main effect of Flanker, $F(1,15)=50.4, p<0.001$.
Latencies were $50 \mathrm{~ms}$ longer on Altering-flanker trials than on Fixed-flanker ones. Flanker also interacted significantly with Instruction, $F(1,15)=12.2, p<0.01$. As can be seen in Fig. 4, the effect of the altering flankers was substantially larger under the parallel (center panels) than under the serial instruction (left panels).

Fixed flanker The data for the Fixed-flanker trials (see Fig. 4, upper panels) were entered into a three-factor ANOVA on the factors Instruction (serial, or parallel), Congruency (congruent, or incongruent), and First-color interval $(50,150$, or $350 \mathrm{~ms})$. Instruction produced a significant main effect, $F(1,15)=20.6, p<0.001$. Latencies were shorter under the serial than under the parallel Instruction (634 vs. $802 \mathrm{~ms}$ ). The analysis revealed also a significant main effect of Congruency (48 ms), $F(1,15)=$ $10.2, p<0.01$.

Furthermore, the two-way interaction between Instruction and Congruency was significant, ${ }^{5} F(1,15)=3.4$, $p=0.083$. The FCE was, on average, $27 \mathrm{~ms}$ in blocks with a serial instruction, and $68 \mathrm{~ms}$ in blocks with a parallel instruction. Further tests revealed that, although Instruction had a larger effect in the incongruent condition, its effect was also significant in the congruent condition, $t(15)=3.90$, $p<0.01$. Apart from that, the effect of First-color interval was significant, $F(2,30)=12.8, p<0.001$. Latencies increased the longer the First-part interval $(696,714$, and $745 \mathrm{~ms}$ ). Finally, there was a significant two-way interaction between Congruency and First-color interval, $F(2,30)=$ $3.52, p<0.05$. The congruency effects were 23,58 , and $62 \mathrm{~ms}$ for the three First-part intervals, respectively.

Altering flanker The data for the Altering-flanker trials (see Fig. 4, lower panels) were entered into a fourfactor ANOVA on the factors Instruction (serial, or parallel), First-part congruency (congruent, or incongruent),

\footnotetext{
5 Since our hypothesis corresponded to a one-tailed statistical test, and the $F$ test is two-tailed, the result can be considered as significant.
} 
Fig. 4 RTI data for Experiments $2 \mathrm{~A}$ and $2 \mathrm{~B}$. The upper panels show the data of the Fixed-flanker trials. The lower panels depict the data of the Altering-flanker trials, in dependence of First-part and Second-part flanker congruency. "Con" and "inc" denote congruent and incongruent, respectively. The congruency effects of the Second-part flankers are expressed by the differences between the filled symbols (congruent) and unfilled symbols (incongruent), whereas the First-part flanker congruency effects (FCEs) can be identified by comparing the different data points with filled, respectively, unfilled symbols in the lower panels
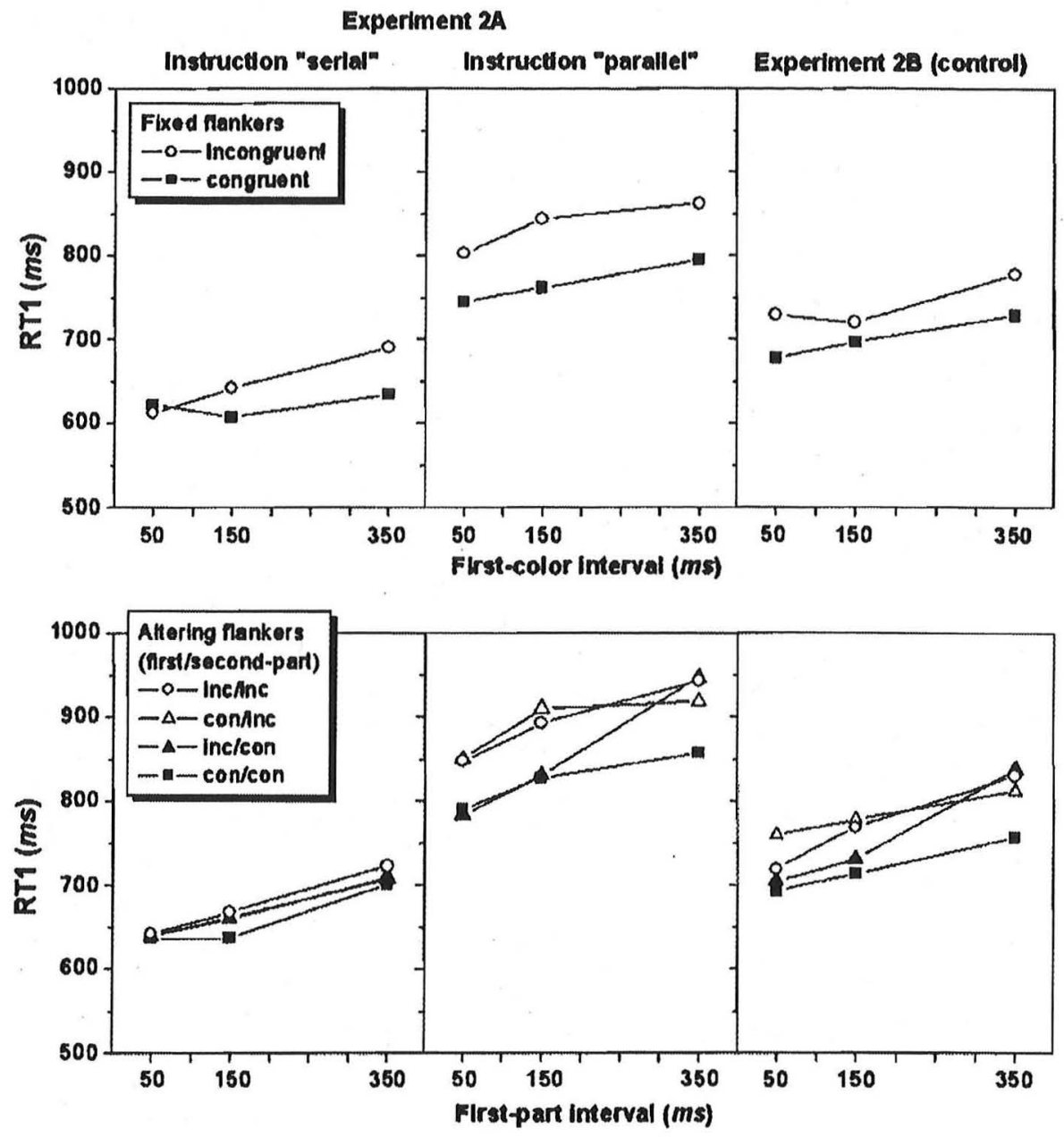

Second-part congruency (congruent, or incongruent), and First-part interval $(50,150$, or $350 \mathrm{~ms})$. First-part congruency had a significant ${ }^{5}$ main effect $(13 \mathrm{~ms}), F(1,15)=$ 3.64, $p=0.076$. Furthermore, the effect of First-part interval was reliable, $F(2,30)=26.8, p<0.001$. Latencies increased by $84 \mathrm{~ms}$ over the First-part interval range. However, First-part congruency interacted significantly ${ }^{5}$ with First-part interval, $F(2,30)=2.68, p=0.085$. The congruency effects were 0,4 , and $35 \mathrm{~ms}$ for the three Firstpart intervals, respectively.

There was also a significant main effect of Instruction, $F(1,15)=27.5, p<0.001$. Latencies under the serial instruction were shorter than those under the parallel instruction ( 669 vs. $866 \mathrm{~ms}$ ). The main effect was further qualified by a reliable three-way interaction between the factors Instruction, First-part congruency and First-part interval, $F(2,30)=9.40, p<0.001$. This interaction indicates that the effects of First-part congruency differed between the serial and the parallel condition mainly at the longest First-part interval (12 vs. $58 \mathrm{~ms}$ ).
Also the main effect of Second-part congruency ( $32 \mathrm{~ms}$ ) was significant, $F(1,15)=10.7, p<0.01$. However, the factor interacted reliably with Instruction, $F(1,15)=12.4$, $p<0.01$. The Second-part congruency effect was smaller under the serial than under the parallel instruction (10 vs. $54 \mathrm{~ms}$ ). As a further test revealed, Instruction had also a significant effect in the congruent condition, $t(15)=4.75$, $p<0.001$.

Neutral flanker The data from the trials with neutral flankers were entered into a two-factor ANOVA with the factors Instruction (serial, or parallel), and First-color interval $(50,150$, or $350 \mathrm{~ms})$. It revealed a significant main effect of Instruction, $F(1,15)=7.26, p<0.05$. As can be seen in Fig. 5, the latencies were $53 \mathrm{~ms}$ longer under the parallel (center panel) than under the serial instruction (left panel). Furthermore, there was a main effect of First-color interval, $F(1,15)=11.4, \quad p<0.001$. The latencies increased by $25 \mathrm{~ms}$ across the First-color intervals.

An ANOVA comparing the data from neutral trials with those from congruent trials with fixed flankers revealed a 
Fig. 5 RTl data for the neutral compared to the congruent condition in Experiments $2 \mathrm{~A}$ and $2 \mathrm{~B}$

\section{Experiment 2A}

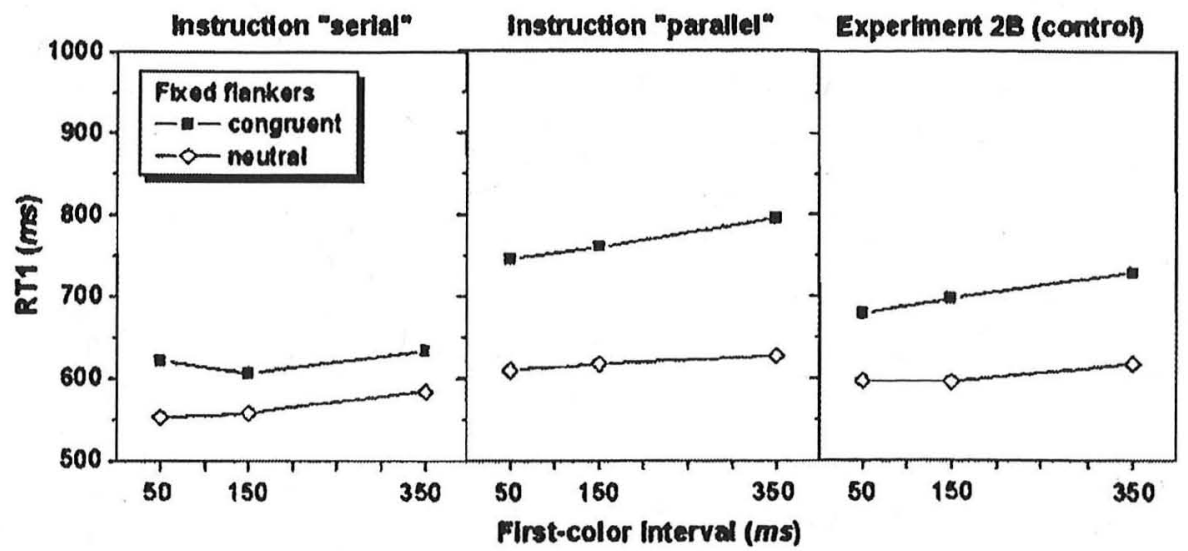

significant main effect of Congruency (congruent, or neutral), $F(1,15)=42.5, p<0.001$. On average, the latencies were $102 \mathrm{~ms}$ longer on congruent trials than on neutral trials. Moreover, Congruency interacted significantly with Instruction, $F(1,15)=12.5, p<0.01$. The difference between the neutral and the congruent condition was higher under the parallel than under the serial instruction (149 vs. $56 \mathrm{~ms}$; see Fig. 5).

$R T 2$ As in the previous experiments, the latencies for the Altering-flanker condition were measured from the onset of the Second-part flankers, but the analysis included the congruency of the First-part flankers as factor.

Fixed flanker Latencies of the correct responses (for R1 and R2) on Fixed-flanker trials (see Fig. 6, upper panels) were entered into a three-factor ANOVA for repeated measures on the factors Instruction (serial, or parallel), Congruency (incongruent, or congruent), and First-color interval $(50,150$, or $350 \mathrm{~ms})$. Congruency produced a significant effect of $73 \mathrm{~ms}, F(1,15)=13.0, p<0.01$. There was no main effect of Instruction, $F(1,15)=1.36$, $p=0.262$. However, Instruction and Congruency interacted significantly, $F(1,15)=6.97, p<0.05$. As can be seen in Fig. 6, the congruency effects were 39 and $108 \mathrm{~ms}$ for the serial (left panels) and the parallel instruction (center panels), respectively. Contrary to RT1, Instruction had a significant effect for the incongruent condition, $t(15)=2.42, p<0.05$, but not for the congruent one, $t(15)=0.054, p=0.957$. Finally, also First-color interval produced a significant effect, $F(2,30)=5.25, p<0.05$. Latencies increased by $33 \mathrm{~ms}$ over the First-color interval range.

Altering flanker The data for correct responses (R1 and R2) of the Altering-flanker trials (see Fig. 6, lower panels) were entered in a four-factor ANOVA for repeated measures on the factors Instruction (serial, or parallel), Firstpart congruency (incongruent, or congruent), Second-part congruency (incongruent, or congruent), and First-part interval $(50,150$, or $350 \mathrm{~ms})$. The analysis revealed a significant main effect of Instruction, $F(1,15)=6.03$, $p<0.05$. RT2 increased from $791 \mathrm{~ms}$ under the serial instruction to $856 \mathrm{~ms}$ under the parallel instruction. The $15 \mathrm{~ms}$ main effect of First-part congruency was significant, ${ }^{5} F(1,15)=3.71, p=0.073$. Furthermore, Secondpart congruency led to a significant effect, $F(1,15)=16.1$, $p<0.01$. Similar to RT1 and also to Experiments $1 \mathrm{~A}$ and $1 \mathrm{~B}$, there was also a reliable interaction between the two congruency factors, $F(1,15)=13.4, p<0.01$. This interaction was strongest at the First-part interval of $350 \mathrm{~ms}$ as the significant three-way interaction between First-part interval and the two congruency factors indicates, $F(2,30)=11.5, p<0.001$.

Second-part congruency also interacted significantly with Instruction, $F(1,15)=25.5, p<0.001$. The congruency effect was substantially smaller under the serial instruction than under the parallel instruction (16 and $95 \mathrm{~ms}$ ). Furthermore, the FCE increased significantly with increasing First-part interval, $F(2,30)=3.30, p<0.05$. Concerning the interaction between Second-part congruency and Instruction, it is important to note that Instruction had a significant effect for the incongruent condition, $t(15)=4.27, p<0.001$, but not for the congruent one, $t(15)=0.834, p=0.417$.

Finally, there was a significant main effect of First-part interval, $F(2,30)=119.0, p<0.001$, which indicates a PRP effect.

\section{Error rates}

The mean error rate for $\mathbf{R} 1$ was $3.17 \%$ under the serial, and $5.46 \%$ under the parallel instruction. In the condition with neutral flankers, the error rate for RI was $5.04 \%$. The error rate for $\mathrm{R} 2$, under the condition that $\mathrm{R} 1$ had also been correct, was $6.63 \%$ under the serial, and $7.61 \%$ under the 
Fig. 6 RT2 data for Experiments $2 \mathrm{~A}$ and $2 \mathrm{~B}$. The upper panels show the data of the Fixed-flanker trials. The lower panels depict the data of the Altering-flanker trials, in dependence of First-part and Second-part flanker congruency. Note that for the Alteringflanker trials, RT2 was measured from the onset of the Second-part flankers. "Con" and "inc" denote congruent and incongruent, respectively. Effects of Second-part flanker congruency are expressed by the differences between the filled symbols (congruent) and unfilled symbols (incongruent), whereas First-part FCEs can be identified by comparing the different data points with filled, respectively, unfilled symbols in the lower panels

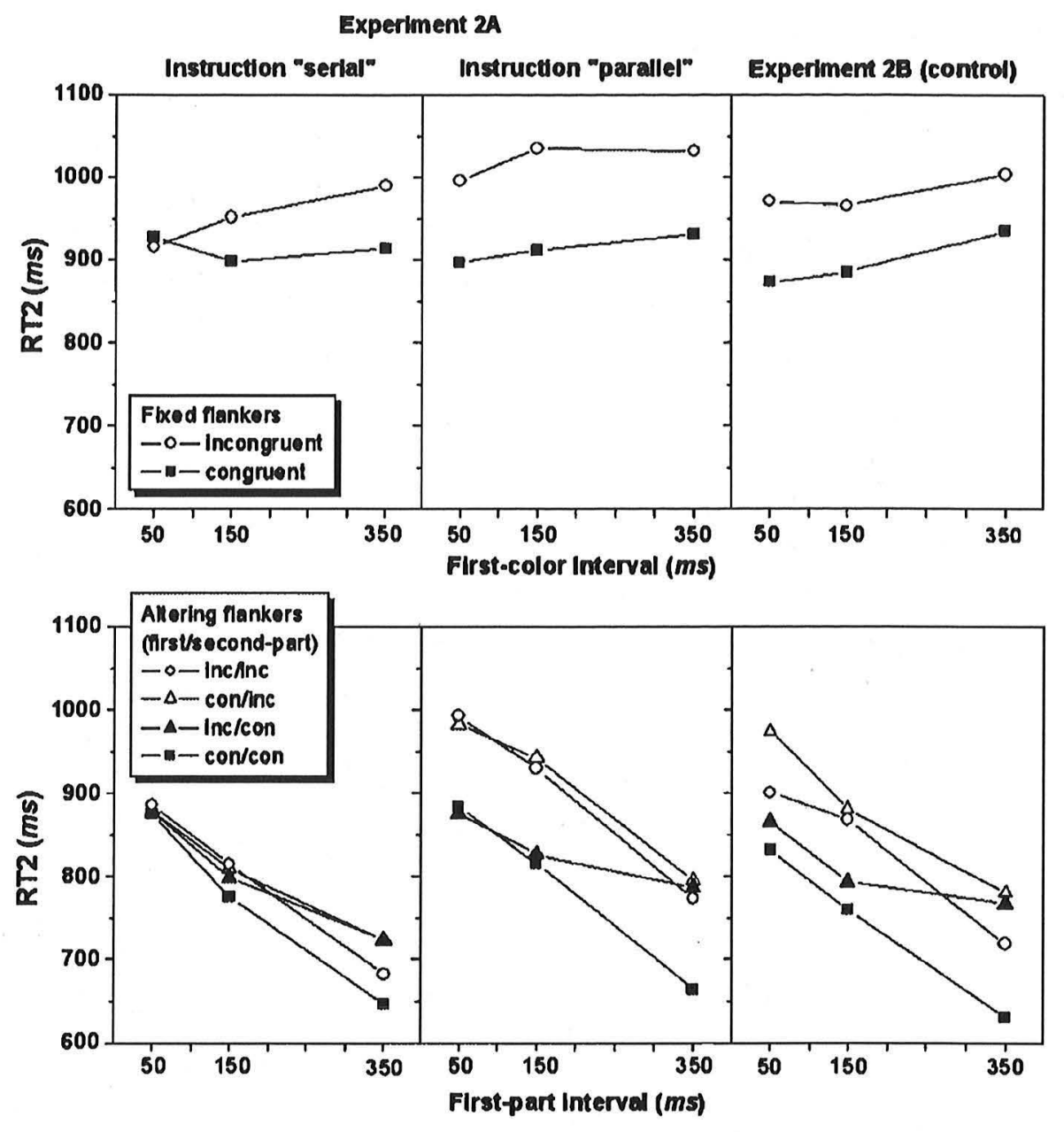

parallel instruction. Because the error rates were again rather low and did not indicate any speed-accuracy tradeoff, they are not further reported here.

\section{Experiment 2B}

Experiment 2B should serve as control condition for Experiment 2A. Therefore, the same procedure was used as in Experiment 2A, except that no specific processing instruction was given to the participants

\section{Method}

A total of 16 students ( 8 females, 8 males) participated in this study under similar conditions as in the previous experiment. Their age ranged from 22 to 38 years ( $M=26.2$ years). All were right-handed (by self report) and had normal or corrected-to-normal vision. None of the students had participated in previous experiments. The stimuli and procedure were the same as in Experiment $2 \mathrm{~A}$ Here, however, no specific processing (serial vs. parallel) instruction was given. Participants were merely informed about the tasks and the response order.

Results

\section{Response times}

The overall results of the present experiment were less extreme than the serial, respectively, the parallel condition of Experiment 2A. Apart from that, as for Experiment 1B, the data pattern including temporal effects and interactions was very similar to Experiment $1 \mathrm{~A}$. Therefore, only the main results of the present experiment will be reported.

RT1 An overall ANOVA including the correct responses for the Fixed-flanker and the Altering-flanker trials on the factors Flanker (fixed, or altering) and Congruency (congruent, or incongruent) revealed a significant main effect of 
Flanker, $F(1,15)=23.2, p<0.001$ (see Fig. 4, right panels). The latencies on Altering-flanker trials were longer than those on Fixed-flanker trials (759 and $721 \mathrm{~ms}$ ). The main effect of (Second-part) Congruency $(39 \mathrm{~ms})$ was significant, ${ }^{5} F(1,15)=4.27, p=0.056$.

Furthermore, an ANOVA comparing the data from neutral trials with those from congruent Fixed-flanker trials revealed a significant main effect of Congruency (congruent, or neutral), $F(1,15)=20.7, p<0.001$. Latencies were $98 \mathrm{~ms}$ longer on congruent trials than on neutral trials (see Fig. 5, right panel).

RT2 Two separate ANOVAs for repeated measurements were conducted on the Fixed-flanker and Altering-flanker trials. There was a significant effect of Congruency (56 ms) on the Fixed-flanker trials, $F(1,15)=7.36, p<0.05$, and a significant main effect of Second-part congruency (80 ms), $F(1,15)=10.9, p<0.01$, on the Altering-flanker trials (see Fig. 6, right panels).

\section{Error rates}

The mean error rate for $\mathrm{R} 1$ was $4.51 \%$, and that for $\mathrm{R} 2$ was $6.70 \%$, given that R1 had also been correct. For the same reasons as in previous experiments, the data are not further reported.

\section{Discussion of Experiments $2 A$ and $2 B$}

As our results show, despite the individual judgment type for each task, the overall pattern of results was strikingly similar to that of Experiments $1 \mathrm{~A}$ and 1B. Although substantially smaller, the FCE-now based on crosstalk between response categories only-was still more pronounced under the parallel than under the serial instruction. Moreover, RT1 was generally increased under the parallel instruction, relative to the serial instruction. In contrast, RT2 was increased under the parallel instruction only for incongruent, but not for congruent flankers. Furthermore, also the results of the Altering-flanker procedure were similar to those in Experiments $1 \mathrm{~A}$ and 1B. The First-part flankers produced significant congruency effects on RT1 and RT2, which were modulated by the instruction condition. Again, as can be seen in Fig. 7, performance in the control condition (Experiment 2B) without specific instruction was again intermediate to those for the serial and parallel instructions (Experiment 2A).

Unlike the previous experiments, flankers that were not assigned to any response were presented on some trials in the present experiments. On these single-task trials, the responses were faster than for congruent flankers. Moreover, this effect was larger under the parallel than under the serial instruction, and intermediate in the control condition. Because the neutral flankers were not related to any response, this effect is probably due to the activation of a different task set for the flankers-CONSONANT/ VOWEL versus of ODD/EVEN number judging-on congruent trials (Rogers \& Monsell, 1995; Steinhauser \& Hübner, 2007; Waszak et al., 2003). This indicates that by parallel processing, not only congruency effects between response categories are increased, but also the conflict between the different task sets seems to be intensified.

\section{General discussion}

In the present study, participants were instructed to vary the degree of parallel processing between dual tasks that were a combination of the Eriksen Flanker task and the PRP paradigm (Telford, 1931). Thus, the costs and benefits of serial and parallel processing could be investigated in two tasks with the same task set (Experiments 1A and 1B) and with different task sets (Experiments 2A and 2B). Our
Fig. 7 RT1 and RT2 data from Experiment $2 \mathrm{~A}$ (serial, parallel) and Experiment $2 \mathrm{~B}$ (neutral). ** $p<0.01, * * * p<0.001$. Al] Fixed- and Altering-flanker trials are included. The RT2 data are shown as measured from onset of SI
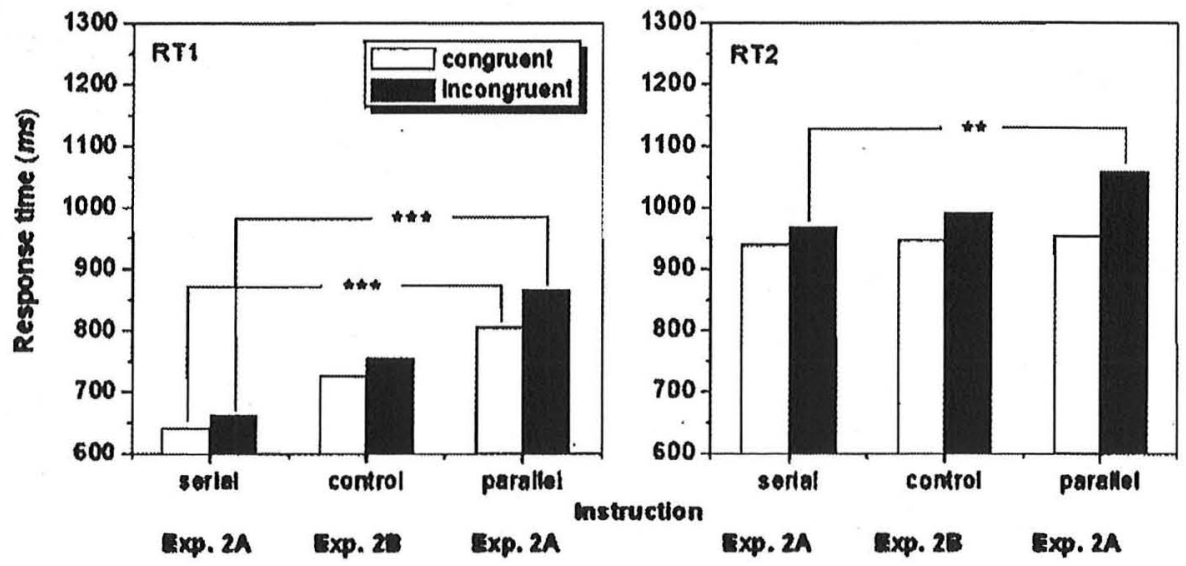
results indicated that the participants were indeed able to vary the degree of parallel processing substantially according to the instruction.

Within each session of the experiments with instruction (Experiments 1A and 2A), participants had to alter between a block-wise serial and a parallel processing strategy. It seems that they could switch immediately between the one and the other processing strategy, because there was no continuous increase or decrease in the degree of parallel processing within each experimental block. ${ }^{3}$ This signals that the strategies we investigated were cognitively accessible and could be selected in a flexible manner, which contrasts to other forms of strategic adjustment in (dual) tasks, such as setting speed versus accuracy (see Strayer \& Kramer, 1994). In the control experiments with no specific instruction (Experiments $1 \mathrm{~B}$ and $2 \mathrm{~B}$ ), an intermediate strategy was applied as compared to the serial and the parallel condition. However, this trend to increased parallel processing compared to the serial instruction in the control experiments underpins our previous observation that, in dual tasks, a moderate parallel processing strategy is preferred (Hübner \& Lehle, 2007).

Furthermore, in order to investigate the strategic modulation within a trial, our procedure contained a specific temporal variation. On half of the trials, initially appearing flankers (First-part flankers) were replaced by new flankers (Second-part flankers) after a short time interval of varying length. Only the Second-part flankers, which were also indicated by a different stimulus color, were relevant for the task. The First-part flankers were presented only shortly and never had to be responded to, so that there was no need for the participants to determine their identity or to coprocess them. Moreover, the participants could have ignored the First-part flankers by a simple filter strategy (i.e., by "attend only to the colored flankers").

Nevertheless, the First-part flankers also had an effect on performance. They produced reliable congruency effects on RT1 as well as on RT2. The degree of their coprocessing was also modified according to the instruction: The effects of the First-part flankers were higher under the parallel instruction compared to the serial instruction or the control condition. Obviously, the participants adjusted the degree of parallel processing by the time the flankers were presented. After that, the processing was not further modified. That the degree of parallel processing remained relatively constant on a trial is in accord with what we found in our previous study (Huibner \& Lehle, 2007).

The main purpose the present study was to compare the costs and benefits of different processing strategies. Since we found a substantial variation in the degree of parallel processing in tasks with the same and with different task sets, such an evaluation is now possible. Altogether, our results clearly demonstrate that there is no benefit of increased parallel processing in dual tasks. Rather, parallel processing repeatedly led to large costs in RT1, whereas RT2 was not affected (in the congruent condition) or also increased (in the incongruent condition). With respect to overt performance, one can conclude that serial processing is the optimal processing strategy in dual tasks. Nevertheless, if not instructed otherwise (see Experiments IB and $2 \mathrm{~B}$ ), participants preferred a moderate parallel processing strategy and thus accepted the costs due to the increased coprocessing. As already pointed out, there was even a tendency of coprocessing the First-part flankers, although they were completely irrelevant for the tasks and although this led to additional costs. How this bias towards parallel processing can be explained is discussed further below.

Because we found a strategic variation in the degree of parallel processing in the present study, we could also comply with another goal, that is, provide an extended version of the CCS model and then evaluate it by a fit to our data. The main idea for the modification of the CCS model was that incongruent flankers produce response conflicts, which have to be resolved by means of cognitive control mechanisms (e.g., Lavie, Hirst, De Fockert, \& Viding, 2004). Because increased levels of cognitive control lead to a higher depletion of central capacity, it can be assumed that the overall capacity available for task processing is reduced in conditions with incongruent compared to congruent flankers. Moreover, it was supposed that the capacity required by control processes increases with the degree of parallel processing. As can be seen in Fig, 8, with these additional assumptions the CCS could be fitted to the data very well (see "Appendix 2" for more details). For the general reduction of central capacity because of the response conflicts, we introduced a new parameter $(z)$ in the model.

The data points in Fig. 8 are arranged in a way that the slope of the functions reflects the increase in RT with the instruction, i.e., with the assumed degree of capacity sharing. For congruent stimuli, RT1 increased with the degree of capacity sharing, whereas the function of RT2 is flat; this is compatible with the original CCS model. In contrast, for incongruent stimuli, RT1 as well as RT2 increased with the degree of capacity sharing irrespective of flanker type and SOA. For fitting these data, the extended version of the CCS model was required. A favorable by-product of the fitting procedure was that the estimates for the degree of capacity sharing in the three instruction conditions were obtained. These values indicated that about $87 \%$ of the central capacity was allocated to Task I under the serial instruction, but only $67 \%$ under the parallel instruction (Experiment 1A). If no specific instruction was given (Experiment 1B), about $75 \%$ of the capacity was allocated to Task 1. 
Fig. 8 In this figure the symbols represent the RTI and RT2 data of Experiments 1A (serial and parallel) and Experiment 1B (control). a The Fixed-flanker trials, b-d The data from the Altering-flanker trials separately for the threeFirst-part intervals $($ b $50 \mathrm{~ms}$, c $150 \mathrm{~ms}$, d $350 \mathrm{~ms}$ ). The lines represent the fit obtained by the model (for details see the text). In the legends "con" and "inc" denote congruent and incongruent, respectively
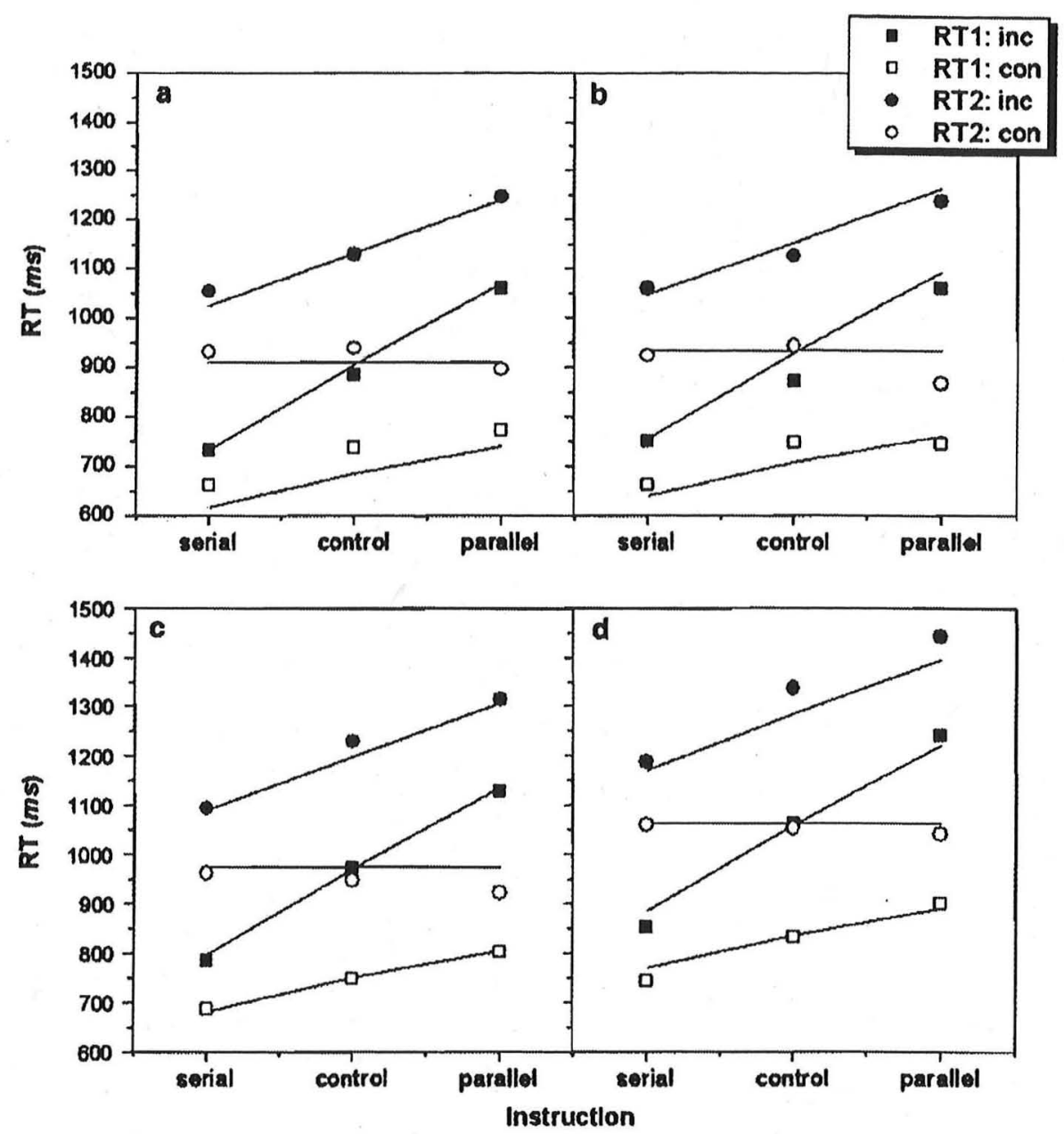

Altogether, a formal model based on the assumption that central capacity is limited in combination with the hypothesis that also conflict solution requires a particular amount of this capacity can explain the present results in a reasonable and parsimonious way. This is also in line with more recent evidence that not only response selection, but also other operations requiring higher cognitive control are limited in dual tasks, e.g., short-term memory consolidation (e.g., Brisson \& Jolicoeur, 2007; Jolicoeur \& Dell'Acqua, 1998), mental rotation (Band \& Miller, 1997), and probably also difficult conditions of stimulus selection (e.g., Jolicoeur, Sessa, Dell'Acqua, \& Robitaille, 2006; Magen \& Cohen, 2005).

A stricter variant of the central capacity limitation idea is the CB model, which assumes that, on the central processing stage, capacity sharing between two tasks is never possible. The modified CB model accounts for crosstalk effects in dual tasks while keeping the hypothesis of a strict bottleneck at response selection (Hommel, 1998; Lien \& Proctor, 2002; Lien et al., 2003). Can this account also explain the present results? In the modified CB model, it is postulated that congruency effects are based on an automatic stimulus-response translation process for each task, which does not require attention or access to limited capacity. Thus, the degree of the congruency effects should merely depend on the time available for the automatic stimulus-response translation process. Accordingly, the explanation according to the modified $\mathrm{CB}$ model would be as follows: the participants have misunderstood the instruction to process the tasks in parallel and merely delayed the first response-possibly with a strategy of response grouping - so that that there was more time available for $S 2$ to produce its automatic effects on RT1.

To examine the possibility of delaying the first response, i.e., to group it together with the execution of the second response, the interresponse-intervals (IRIs) are sometimes analyzed (Miller \& Alderton, 2006; Pashler, 1994b). Short IRIs are believed to indicate that frequent response grouping occurred in a task. However, it has to be noted 
that the CCS model also predicts such a decrease with increased capacity sharing. This results from the fact that RTl increases with an increased degree of capacity sharing, whereas RT2 remains unaffected. Thus, analyzing the IRIs does not help to reveal a strategy of mere delay versus capacity sharing.

If the effects of the instruction we found in the present study were exclusively based on more delay of the first response without increased coprocessing under the parallel condition, then the large effects on RT2 had also to be explained by a delay of the first response. However, as has been shown, RT2 is only marginally influenced by a strategy of response grouping (Pashler \& Johnston, 1989; Ruthruff, Pashler, \& Klaassen, 2001). The situation is even more complicated because, in the present study, RT2 was significantly increased only in the incongruent condition. This would mean that the delay in the first response is carried forward to RT2 only in the incompatible, but not in the compatible condition, which is implausible.

Moreover, also the irrelevant First-part flankers produced congruency effects although they never had to be responded to. Correspondingly, their effects cannot be attributed to response grouping or response delay. Furthermore, in contrast to the Second-part flankers, the Firstpart flankers were presented for a limited time only which was independent of the response times. The automatic activation hypothesis in the modified $\mathrm{CB}$ model would therefore predict equal effects of the First-part flankers with the serial and the parallel instruction condition. However, the First-part flanker coprocessing led to larger overall costs and congruency effects under the parallel compared to the serial instruction condition. Thus, altogether, the present pattern of results can be more plausibly explained by assuming crosstalk based on strategic compared to automatic effects. That the FCE in dual tasks reflects the degree of strategic rather than automatic parallel processing was also demonstrated by previous studies (Hübner \& Lehle, 2007; Lehle \& Hübner, 2008).

Apart from the CB and the CCS model, which are rather similar to each other in many respects, other classes of models have been proposed that neither assume a $\mathrm{CB}$ nor a central capacity limitation, but a strategic allocation of capacity or attention between two tasks. Among these is the strategic response deferment (SRD) model of Meyer \& Kieras (1997), which postulates that strategies play a major role in dual task results. However, it is also assumed that perfect time sharing can be realized. This means that it should be possible to eliminate dual-task costs such as the PRP effect or costs on RT1 completely by a strategy of parallel processing. However, as considerable costs of parallel processing were found in our experiments that even increased with increased parallel processing, the SRD model can hardly account for our data.
Another dual-task model accounting for the influence of strategic allocation of attention is the executive control theory of visual attention (ECTVA) (Logan \& Gordon, 2001). This model is based on evidence accumulation and includes mechanisms for producing crosstalk between stimulus categories. However, there are two main results in the present experiments that cannot be explained by the model. First, because the model does not distinguish between response categories and stimulus categories, it does not predict the congruency effects in Experiments $2 \mathrm{~A}$ and 2B (see also Huibner \& Druey, 2006; Watter \& Logan, 2006). Furthermore, the ECTVA assumes that the degree of parallel processing is controlled by a mechanism of selective attention. Because this mechanism is also supposed to be responsible for feature integration, increased parallel processing should lead to an extremely high error rate in case of incongruent stimuli. In the present experiments, however, the error rates were hardly affected by an intensive capacity sharing, so that the parallel processing we observed does certainly not imply a responding before target and flankers were identified.

However, before final conclusions can be drawn, the question has to be clarified why people obviously prefer a rather parallel processing strategy-if not instructed otherwise--even if there is no benefit in overt performance. One can hypothesize that a serial strategy in PRP-like dual tasks requires more cognitive control compared to a parallel strategy: During the first task, serial processing requires a strict focusing of attention on S1 and the inhibition of S2. processing. To conduct R2 subsequently, the inhibition of $\mathrm{S} 2$ has to be abolished and S2 has to be processed. That the processing of previously inhibited stimuli requires effortful control has been shown in studies of Negative Priming (e.g., Tipper, 1985; Tipper \& Cranston, I985) and Inhibition of Return (e.g., Posner \& Cohen, 1984; Tipper, Weaver, Jerreat, \& Burak, 1994).

In order to prevent a strenuous processing of inhibited flankers, the participants might have a tendency to process the target and flankers in parallel right from the beginning of a trial and to keep the degree of capacity sharing constant. This could also explain why the irrelevant First-part flankers were coprocessed to the same degree. The hypothesis that serial processing is more effortful in PRP. like dual tasks is supported by a recent study where we demonstrated that serial processing is accompanied by an increased heart rate and by higher levels of subjective effort compared to parallel processing (Lehle, Steinhauser, \& Hübner, 2008).

To conclude, the present study demonstrates that participants have a tendency to moderate parallel processing in dual tasks, although they are able to switch between serial and parallel processing quite flexibly and although parallel processing leads to considerable costs in performance. The 
present pattern of results can be reasonably explained by assuming a strategic allocation of central capacity in combination with increased capacity demands in situations with incongruent stimuli. Future research should address the role of processing strategies in dual tasks further, since little is known about strategic effects in dual tasks and also other cognitive paradigms. Furthermore, there is a need for theoretical clarification in this respect. Attempts should be undertaken to further evaluate and possibly integrate the diverse theoretical accounts that have been proposed in order to explain performance in dual tasks.

Acknowledgments We thank Peter Frensch and two anonymous reviewers for their valuable comments on a former version of the manuscript.

\section{Appendix 1}

Formal description of the CCS model

In this appendix we briefly describe the CCS model in formal terms. The formalization is important to understand how we modified the model to fit it to the data of Experiments $1 \mathrm{~A}$ and $1 \mathrm{~B}$, which is described in "Appendix 2 ". In Fig. 9, which shows typical PRP situations (they correspond to type B in Tombu \& Jolicoeur, 2003), the assumed processing stages are represented by areas, whose size corresponds to the work $(W)$ necessary to accomplish the processing at the respective stage. $W$ is measured in units of capacity (c). Unless the task as such is modified, the work necessary for a given task at a stage is fixed. The vertical extensions of the areas correspond to the momentary processing rate $r$, which can vary between 0 and the maximum rate $r_{\max }$. Usually, and without loss of generality, the maximum rate can be set to 1 . The unit of $r$ is capacity per second $(\mathrm{c} / \mathrm{s})$.

Because it is assumed that the stages $\mathrm{A}$ and $\mathrm{C}$ have unlimited capacity, the height of their corresponding areas is constantly $r_{\max }$. Therefore, the duration of these stages can simply be calculated by dividing the necessary work by the maximum rate (i.e., $T=W / r_{\max }$ ). More specifically, for two tasks, we have as processing times for the stages Al, $\mathrm{C} 1, \quad \mathrm{~A} 2$, and $\mathrm{C} 2: T_{\mathrm{Al}}=W_{\mathrm{Al}} / r_{\text {max }}, \quad T_{\mathrm{Cl}}=W_{\mathrm{Cl}} / r_{\text {max }}$, $T_{\mathrm{A} 2}=W_{\mathrm{A} 2} / r_{\text {max }}$, and $T_{\mathrm{C} 2}=W_{\mathrm{C} 2} / r_{\text {max }}$, respectively. Interpreted in graphical terms, this means that the horizontal extensions of the areas in Fig. 9 directly reflect their relative duration of processing. The crucial point is to calculate the duration for the stages B1 and B2. According to the CCS model, the capacity for the central stage is limited, but can be divided between the tasks.

During the overlap of the central stages, the capacity limit implies that the processing rates for $\mathrm{B} 1$ and $\mathrm{B} 2$ have to sum up to $r_{\max }$ at each point in time. Let us denote the

\section{Short SOA}

a $\$ 1$
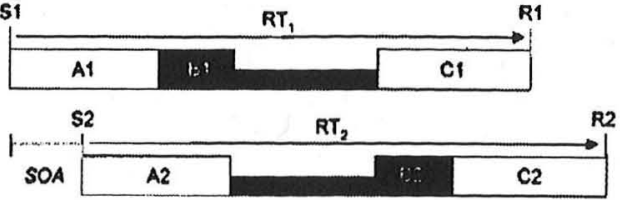

b s1
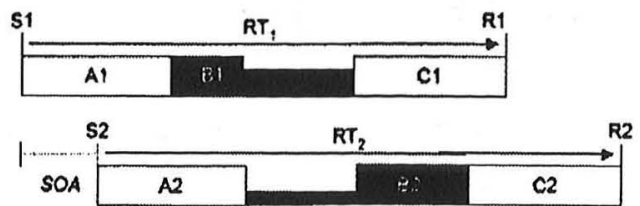

c $\mathbf{s}$

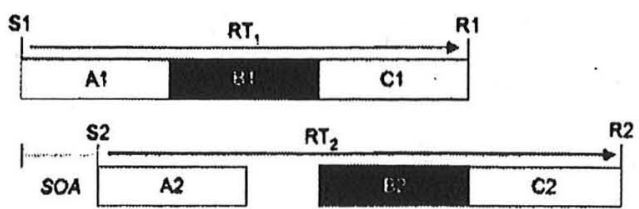

II. Long SOA
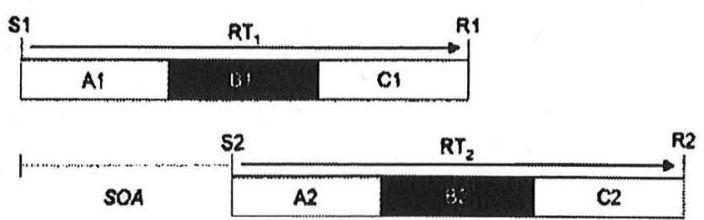

Fig. 9 Predictions of the CCS model are illustrated at short $(I)$ and long SOA $(I I)$. Areas $A /$ and $A 2$ represent the perceptual stages, whereas the areas $C l$ and $C 2$ refer to the motor execution stages for Task 1 and Task 2, respectively. $B /$ and $B 2$ denote the corresponding central stages with limited capacity. I a Central capacity is equally distributed between Task $I$ and Task 2 ; I b more capacity is allocated on Task $1.1 \mathrm{c}$ The $\mathrm{CB}$ situation where all capacity is concentrated on Task 1. RT1 increases the more of the central capacity is allocated to Task 2 (compare I a-c). RT2 decreases with increasing SOA (compare I and II), whereas it is independent from the degree of capacity sharing (compare I a-c)

rate for stage $\mathrm{B} 1$ at time $t$ by $r_{\mathrm{B} 1}(t)$. Then, the rate for $\mathrm{B} 2$ at that time is $r_{\max }-r_{\mathrm{BI}}(t)$. Consequently, the durations $T_{\mathrm{BI}}$ and $T_{\mathrm{B} 2}$ depend on the central overlap between the tasks and on the degree of capacity sharing. If we consider the special case where the rate for $\mathrm{Bl}$ is constantly $r_{\max }$, then the situation corresponds to a strict $\mathrm{CB}$. Therefore, all results supporting a $\mathrm{CB}$ can also be accounted for by the CCS model. Additionally, however, the CCS model makes specific predictions for situations in which capacity is shared between the central stages. Two such situations with different sharing proportions are shown in Fig. 9.

$T_{\mathrm{B} 1}$ and $T_{\mathrm{B} 2}$ can be computed by piecewise calculations. As can be seen in Fig. 9, the temporal overlap between B1 and $\mathrm{B} 2$ depends on the difference $T_{\mathrm{A} 2}-T_{\mathrm{A} 1}$ between the durations of the stages $\mathrm{Al}$ and $\mathrm{A} 2$, and on the SOA. Obviously, in the interval $\mathrm{SOA}+T_{\mathrm{A} 2}-T_{\mathrm{A} 1}$, the 
processing rate for $\mathrm{B} 1$ equals $r_{\max }$. The work done in this interval is $\left(\mathrm{SOA}+T_{\mathrm{A} 2}-T_{\mathrm{A} 1}\right) \cdot r_{\max }$. The remaining work $\left[W_{\mathrm{B} 1}-\left(\mathrm{SOA}+T_{\mathrm{A} 2}-T_{\mathrm{A} 1}\right) \cdot r_{\text {max }}\right]$ is subsequently completed with a rate of $r_{\mathrm{B} 1}$. Thus, taken together, $T_{\mathrm{B} \text { I }}$ can be computed as

$$
\begin{aligned}
T_{\mathrm{B} 1}= & \mathrm{SOA}+T_{\mathrm{A} 2}-T_{\mathrm{A} 1} \\
& +\left[W_{\mathrm{B} 1}-\left(\mathrm{SOA}+T_{\mathrm{A} 2}-T_{\mathrm{Al}}\right) \cdot r_{\max }\right] / r_{\mathrm{B} 1} .
\end{aligned}
$$

In graphical terms, $T_{\mathrm{B} 1}$ is reflected by the length of area B1 (see Fig. 9). An important prediction can be derived by Eq. 1. Because the work for Bl is fixed, the duration (length) of this stage depends on the processing rate (height). RTl is thus predicted to increase with a decreasing rate. That is, the more capacity is shared with B2, the more costs are produced for RT1. The duration of $\mathrm{B} 2$ is calculated analogously to that of $\mathrm{B} 1$ :

$$
\begin{aligned}
T_{\mathrm{B} 2}= & {\left[W_{\mathrm{B} 1}-\left(\mathrm{SOA}+T_{\mathrm{A} 2}-T_{\mathrm{A} 1}\right) \cdot r_{\max }\right] / r_{\mathrm{B} 1} } \\
& +\left(W_{\mathrm{B} 2}-\left[W_{\mathrm{B} 1}-\left(\mathrm{SOA}+T_{\mathrm{A} 2}-T_{\mathrm{A} 1}\right) \cdot r_{\max }\right]\right. \\
& \left./ r_{\mathrm{B} 1} \cdot r_{\mathrm{B} 2}\right) / r_{\max } \\
= & T_{\mathrm{A} 1}-T_{\mathrm{A} 2}-\mathrm{SOA}+W_{\mathrm{B} 1} / r_{\max }+W_{\mathrm{B} 2} / r_{\max } .
\end{aligned}
$$

If we consider Eq. 2, then it is obvious that the dependence of $T_{\mathrm{B} 2}$ on the SOA explains the PRP effect. Apart from that, $T_{\mathrm{B} 2}$ depends on the work required for the processing of Task 1 and Task 2. However, $T_{\mathrm{B} 2}$ does not depend on the relative rates (see Fig. 9). This property of the model implies that $T_{\mathrm{B} 2}$ and, thus, also RT2 is not affected by the degree of capacity sharing. In other words, sharing capacity with Task 2 does not produce any benefit on RT2, but it produces costs on RT1. Therefore, according to the CCS model, a strict serial processing strategy would be optimal. Furthermore, RT1 is predicted to decrease with an increasing SOA. Moreover, this effect should be the stronger, the smaller $r_{\mathrm{B} 1}$. Only if $r_{\mathrm{B}}$ equals $r_{\max }$, then no influence of SOA on the performance for Task 1 should be observed.

\section{Appendix 2}

Fitting the CCS model to the data

The model was fitted to 48 mean data points from the Experiments $1 \mathrm{~A}$ and $1 \mathrm{~B}$. Twelve of these points were from the Fixed-flanker condition: six points for RT1, and six points for RT2. The six points for each response type represent the three instructions and the two congruency conditions (the small first-color effect was ignored). The other 36 data points were taken from the Altering-flanker condition. The data pattern was the same as for the Fixedflanker condition. However, there was one pattern for each of the three First-part intervals. Furthermore, only those trials were included in which the congruency type of the First-part flanker was the same as that of the Second-part flanker.

The use of the same judgment type for both tasks might be problematic in some respect. For fitting the data to a formal model, however, this condition is favorable, because it needs only a relatively small number of free parameters for its description. This is a crucial prerequisite in view of our restricted number of data points. We started with a formal version of the standard CCS model (see "Appendix 1"), and considered congruent trials as the standard situation for this version. We then extended the model to also account for the performance on incongruent trials, where we considered only the Fixed-flanker trials and those Altering-flanker trials, where the congruency type did not change between the First-part flankers and the Second-part flankers.

Because the same judgment types were used for both tasks in Experiment $1 \mathrm{~A}$ and $1 \mathrm{~B}$, we made the reasonable assumption that the processing of Task 1 required the same work as that of Task 2 . It follows that $T_{\mathrm{A} 1}=T_{\mathrm{A} 2}$, and $T_{\mathrm{C} 1}=T_{\mathrm{C} 2}$. Furthermore, as there was no variation in the duration of perceptual processing or in the execution of responses, $W_{\mathrm{A}}$ and $W_{\mathrm{C}}$ were considered as constant for all conditions. Apart from that, we assumed that capacity was shared in such a way that $T_{\mathrm{B} 1}$ was always less or equal to $T_{\mathrm{B} 2}$. In other words, less capacity was allocated to $\mathrm{S} 2$ than to $\mathrm{S} 1$ during the processing of Task 1.

The deficit of the standard CCS model with respect to the present experiments is that it cannot account for congruency effects. Therefore, we added the following assumptions to the model: first, also the crosstalk between the tasks consumes part of the central capacity. However, we supposed that for congruent stimuli the costs are outweighed by the positive response priming. Thus, the congruent situation was considered as functionally equivalent to the standard situation. For incongruent stimuli, though, the situation is different. They produce negative response priming, so that there is no compensation for the capacity reduction. Thus, for incongruent stimuli, we had to implement a reduction of central capacity. This was modeled by multiplying the processing rates $r_{\mathrm{B} 1}$ and $r_{\mathrm{B} 2}$ by a common reduction parameter $z$, which could vary between 0.5 and 1 .

To show this in more detail, let us first consider the Fixed-flanker condition. Because we had a simultaneous onset of the stimuli in this case, the equations are relatively simple (the small First-color effect is ignored). Altogether, with our assumptions, Eq. 1 is modified in Eq. 3:

$T_{\mathrm{B} 1}=\left[1 /\left(r_{\mathrm{B} 1} \cdot z\right)\right] \cdot W_{\mathrm{BI}}$.

A preliminary attempt to fit the model to the data confirmed our suspicion that the reduction of capacity was 
not constant but depended on the degree of capacity sharing. With a constant $z$, no satisfactory results were obtained for RT2. Presumably, this reflects the mechanism that an increased sharing leads to an increased crosstalk, which, in turn, consumes more central capacity. To take this into account, and to limit the number of extra parameters, $z$ was defined as a linear function of $r_{\mathrm{B}}$, i.e.: $z=\mathrm{d} \cdot r_{\mathrm{B} /} / r_{\max }+e$, where $d$ and $e$ are free parameters.

The duration of stage $\mathrm{B} 2$ is determined by the length of the central overlap interval between the tasks, [1/ $\left.\left.\left(r_{\mathrm{B} 1} \cdot z\right)\right] \cdot W_{\mathrm{B} 1}\right]$, and by the time needed for the remaining work $\left(W_{\mathrm{B} 2}-\left[1 /\left(r_{\mathrm{B} 1} \cdot z\right)\right] \cdot W_{\mathrm{B} 1} \cdot r_{\mathrm{B} 2} \cdot z\right) \cdot r_{\max }$. Taken together, we have in Eq. 4:

$\begin{aligned} T_{\mathrm{B} 2}= & {\left[1 /\left(r_{\mathrm{B} 1} \cdot z\right)\right] \cdot W_{\mathrm{B} 1} } \\ & +\left(W_{\mathrm{B} 2}-\left[1 /\left(r_{\mathrm{B} 1} \cdot z\right)\right] \cdot W_{\mathrm{B} 1} \cdot r_{\mathrm{B} 2} \cdot z\right) / r_{\max } .\end{aligned}$

Simplification leads to

$T_{\mathrm{B} 2}=W_{\mathrm{B} 1} \cdot\left(1 / r_{\mathrm{B} 1}\right)(1 / z-1)+W_{\mathrm{B} 1} / r_{\max }+W_{\mathrm{B} 2} / r_{\max }$.

As can be seen, if $z=1$, as is assumed for congruent stimuli, we have the same results for $T_{\mathrm{B} 1}$ and $T_{\mathrm{B} 2}$ as in Eqs. 1 and 2 . If capacity is reduced, however, response times are increased. Because $z$ is also part of the equation for $T_{\mathrm{B} 2}$, RT2 increases with the degree of capacity sharing, in contrast to Eq. 2.

These basic equations also apply to the Altering-flanker condition. However, because the results are relatively complicated for the condition where the congruency type changed from the First-part to the Second-part flankers, we included only the trials in which First-part and Second-part flankers were of the same congruency type. For these trials, the performance was always rather similar to that in the corresponding Fixed-flanker conditions. We merely had to take the First-part interval variation into account. Our results show that the change of flanker identity on these trials produced some costs, which increased with increased duration of the First-part interval. Therefore, we modeled the Altering-flanker data in the same way as the Fixedflanker data, except that the term ' $g$. INT' (INT $=$ interval), was added to the equations. This term increases the time for the central stages in Eq. 5, as compared to Eqs. 3 and 4 for the Fixed-flanker trials, by some duration that is proportional to the First-part interval:

$$
\begin{aligned}
T_{\mathrm{B} 1}= & {\left[1 /\left(r_{\mathrm{B} 1} \cdot z\right)\right] \cdot W_{\mathrm{B} 1}+g \cdot \mathrm{INT} . } \\
T_{\mathrm{B} 2}= & W_{\mathrm{B} 1} \cdot\left(1 / r_{\mathrm{B} 1}\right)(1 / z-1)+W_{\mathrm{B} 1} \cdot(\mathrm{s} / \mathrm{c})+W_{\mathrm{B} 2} \cdot(\mathrm{s} / \mathrm{c}) \\
& +g \cdot \text { INT. }
\end{aligned}
$$

The work for the central stages, $W_{\mathrm{B} 1}$ and $W_{\mathrm{B} 2}$, was deliberately set to $500 \mathrm{c}$, respectively. Because the pre- and post-central stages were assumed to be identical for both tasks and constant for all conditions, they were modeled by a single additive constant. Moreover, because the predicted
RTs had also to be scaled in order to be in the same range as our data, we used a single linear transformation to obtain the estimated RTs, i.e., RT1 $=\mathrm{a} \cdot T_{\mathrm{B} 1}+\mathrm{b}$ and $\mathrm{RT} 2=$ $\mathrm{a} \cdot T_{\mathrm{B} 2}+\mathrm{b}$.

Altogether, we had 6 relevant parameters for fitting the 48 data points: three values for $r_{\mathrm{B}}$ corresponding to the three instruction conditions, two linear parameters $d$ and $e$ for computing the capacity reduction $z$, and one parameter $g$ for the first-part interval effect. The model was fitted by a routine (SIMPLEX) that estimated the parameter values by minimizing the sum of squared errors.

As estimation for $r_{\mathrm{B}}$ the procedure revealed the values $0.872,0.747$, and 0.666 for the serial, neutral, and parallel condition, respectively. The values of the parameters $d$ and $e$ to determine the reduction parameter $z$ were 0.803 and 0.0729 , respectively. The obtained value for parameter $g$ was 0.633 . Finally, the scaling parameters $a$ and $b$ were 0.684 and 226. As can be seen in Fig. 9, with these parameters the model fits the data very well. This is also reflected by the corresponding $\mathrm{R}^{2}$ of 0.999 .

\section{References}

Band, G. P. H., \& Miller, J. (1997). Mental rotation interferes with response preparation. Journal of Experimental Psychology: Human Perception and Performance, 23, 319-338.

Brisson, B., \& Jolicoeur, P. (2007). A psychological refractory period in access to visual short-term memory and the deployment of visual-spatial attention: Multitasking processing deficits revealed by event-related potentials. Psychophysiology, 44, 323-333.

Cohen, A., \& Shoup, R. (1997). Perceptual dimensional constraints in response selection processes. Cognitive Psychology, 32, 128-181.

Ellenbogen, R., \& Meiran, N. (2008). Working memory involvement in dual-task performance: Evidence from the backward compatibility effect. Memory \& Cognition, 36, 968-978.

Fischer, R., Miller, J., \& Schubert, T. (2007). Evidence for parallel semantic memory retrieval in dual tasks. Memory \& Cognition, 35, 1685-1699.

Gopher, D. (1986), In defense of resources: On structures, energies, pools and the allocation of attention. In G. R. J. Hockey, A. W. K. Gaillard \& M. G. H. Coles (Eds.), Energetics and human information processing (pp. 352-371). Dordrecht: Martinus Nijhoff Publishers.

Hommel, B. (1998). Automatic stimulus-response translation in dualtask performance. Journal of Experimental Psychology: Human Perception and Performance, 24, 1368-1384.

Hübner, R., \& Druey, M. D. (2006). Response execution, selection, or activation: What is sufficient for response-related repetition effects under task shifting? Psychological Research, 70, 245-261.

Hübner, R., \& Lehle, C. (2007). Strategies of flanker coprocessing in single and dual tasks. Journal of Experimental Psychology: Human Perception and Performance, 33, I03-123.

Jolicoeur, P., \& Dell'Acqua, R. (1998). The demonstration of shortterm memory consolidation. Cognitive Psychology, 36, 138-202.

Jolicoeur, P., Sessa, P., Dell'Acqua, R., \& Robitaille, N. (2006). On the control of visual spatial attention: Evidence from human electrophysiology. Psychological Research, 70, 414-424. 
Kahneman, D. (1973). Attention and effort. Englewood Cliffs, NJ: Prentice-Hall.

Lavie, N., Hirst, A., De Fockert, J. W., \& Viding, E. (2004). Load theory of selective attention and cognitive control. Journal of Experimental Psychology: General, 133, 339-354.

Lehle, C., \& Hübner, R. (2008). On-the-fly adaptation of selectivity in the flanker task. Psychonomic Bulletin \& Review, 15, 814-818.

Leble, C., Steinhauser, M., \& Hübner, R. (2008). Serial or parallel processing in dual tasks: What is more effortful? Psychophysiology (in press).

Lien, M. C., \& Proctor, R. W. (2002). Stimulus-response compatibility and psychological refractory period effects: Implications for response selection. Psychonomic Bulletin \& Review, 9, 212-238.

Lien, M. C., Schweickert, R., \& Proctor, R. W. (2003). Task switching and response correspondence in the psychological refractory period paradigm. Journal of Experimental Psychology: Human Perception and Performance, 29, 692-712.

Logan, G. D., \& Gordon, R. D. (2001). Executive control of visual attention in dual-task situations. Psychological Review, 108, $393-434$.

Logan, G. D., \& Schulkind, M. D. (2000). Parallel memory retrieval in dual-task situations: 1. Semantic memory. Journal of Experimental Psychology: Human Perception and Performance, 26, 1072-1090.

Magen, H., \& Cohen, A. (2005). Location specificity in response selection processes for visual stimuli. Psychonomic Bulletin \& Review, 12, 541-548.

McLeod, P. (1977). Parallel processing and the psychological refractory period. Acta Psychologica, 41, 381-396.

Meyer, D. E., \& Kieras, D. E. (1997). A computational theory of executive cognitive processes and multiple-task performance: Part 1. Basic mechanisms. Psychological Review, 104, 3-65.

Miller, J. (2006). Backward crosstalk effects in psychological refractory period paradigms: Effects of second-task response types on first-task response latencies. Psychological Research, $70,483-493$.

Miller, J., \& Alderton, M. (2006). Backward response-level crosstalk in the psychological refractory period paradigm. Journal of Experimental Psychology: Human Perception and Performance, $32,149-165$.

Miller, J., Ulrich, R,, \& Rolke, B. (2008). Parallel and serial processing in dual-tasking: An optimization account. Cognitive Psychology (in press).

Navon, D., \& Miller, J. (1987). Role of outcome conflict in dual-task interference. Journal of Experimental Psychology: Human Perception and Performance, 13, 435-448.

Navon, D., \& Miller, J. (2002). Queuing or sharing? A critical evaluation of the single-bottleneck notion. Cognitive Psychology, 44, 193-251.

Oriet, C., Tombu, M., \& Jolicoeur, P. (2005). Symbolic distance affects two processing loci in the number comparison task. Memory \& Cognition, 33, 913-926.

Pashler, H. E. (1984). Processing stages in overlapping tasks: Evidence for a central bottleneck. Joumal of Experimental Psychology: Human Perception and Performance, 10, 358-377.
Pashler, H. E. (1994a). Dual-task interference in simple tasks: Data and theory. Psychological Bulletin, 116, 220-244.

Pashler, H. E. (1994b). Graded capacity-sharing in dual-task interference? Journal of Experimental Psychology: Human Perception and Performance, 20, 330-342.

Pashler, H. E., \& Johnston, J. C. (1989). Chronometric evidence for central postponement in temporally overlapping tasks. Quarterly Journal of Experimental Psychology: Human Experimental Psychology, 4l, 19-45.

Pashler, H. E., Johnston, J. C., \& Ruthruff, E. (2001). Attention and performance. Annual Review of Psychology, 52, 629-651.

Posner, M. I., \& Cohen, Y. (1984). Components of visual orienting. In H. Bouma \& D. G. Bouwhuis (Eds.), Attention and performance $X$ : Control of language processes (Vol. 32, pp. 531-556). London: Lawrence Erlbaum Associates.

Rogers, R. D., \& Monsell, S. (1995). Costs of a predictable switch between simple cognitive tasks. Journal of Experimental Psychology: General, 124, 207-231.

Ruthruff, E., Pashler, H. E., \& Klaassen, A. (2001). Processing bottlenecks in dual-task performance: Structural limitation or strategic postponement? Psychonomic Bulletin \& Review. $8,73-80$.

Steinhauser, M., \& Hübner, R. (2007). Automatic activation of taskrelated representations in task shifting. Memory \& Cognition, 35 . 138-155.

Strayer, D. L., \& Kramer, A. F. (1994). Strategies and automaticity: II Dynamic aspects of strategy adjustment. Journal of Experimen. tal Psychology: Learning, Memory, and Cognition, 20, 342-365.

Stürmer, B., Seiss, E., \& Leuthold, H. (2005). Executive control in the Simon task: A dual-task examination of response priming and its suppression. European Joumal of Cognitive Psychology, 17, 590-618.

Telford, C. W. (1931). The refractory phase of voluntary and associative responses. Journal of Experimental Psychology, 14 $1-36$.

Tipper, S. P. (1985). The negative priming effect: Inhibitory priming by ignored objects. The Quarterly Journal of Experimental Psychology A: Human Experimental Psychology, 37A, 571-590.

Tipper, S. P., \& Cranston, M. (1985). Selective attention and priming: Inhibitory and facilitory effects of ignored primes. Quarterly Journal of Experimental Psychology A: Human Experimental Psychology, 37A, 591-611.

Tipper, S. P., Weaver, B., Jerreat, L. M., \& Burak, A. L. (1994). Object-based and environment-based inhibition of return of visual attention. Journal of Experimental Psychology: Human Perception and Performance, 20, 478-499.

Tombu, M., \& Jolicoeur, P. (2003). A central capacity sharing model of dual-task performance. Journal of Experimental Psychology: Human Perception and Performance, 29, 3-18.

Waszak, F., Hommel, B., \& Allport, A. (2003). Task-switching and long-term priming: Role of episodic stimulus-task bindings in task-shift costs. Cognitive Psychology, 46, 361-413.

Watter, S., \& Logan, G. D. (2006). Parallel response selection in dualtask situations. Perception \& Psychophysics, 68, 254-277. 